\title{
TRIANGULAR GENERALIZED LIE BIALGEBROIDS: HOMOLOGY AND COHOMOLOGY THEORIES
}

\author{
DAVID IGLESIAS ${ }^{1}$, BELEN LOPEZ ${ }^{2}$, \\ JUAN C. MARRERO ${ }^{1}$ and EDITH PADRON ${ }^{1}$ \\ ${ }^{1}$ Departamento de Matemática Fundamental, Facultad de Matemáticas \\ Universidad de la Laguna, La Laguna \\ Tenerife, Canary Islands, Spain \\ E-mail:diglesia@ull.es, jcmarrer@ull.es,mepadron@ull.es \\ ${ }^{2}$ Departamento de Matemáticas, Edificio de Matemáticas e Informática \\ Campus Universitario de Tafira, Universidad de Las Palmas de Gran Canaria \\ 35017 Las Palmas, Canary Islands, Spain \\ E-mail: blopez@dma.ulpgc.es
}

\begin{abstract}
Triangular generalized Lie bialgebroids are a generalization of triangular Lie bialgebroids in the sense of Mackenzie and Xu. For this type of structures two homology and cohomology theories are considered. Moreover, we prove that the vanishing of a certain cohomology class, which we will call the modular class, implies the existence of a duality between these homology and cohomology theories. As a consequence, we recover some previous results for unimodular Poisson and Jacobi manifolds and unimodular triangular Lie bialgebroids.
\end{abstract}

1. Introduction. Lie bialgebroids were introduced and studied by Mackenzie and $\mathrm{Xu}$ [MX1] (see also [Ko1]) as the infinitesimal invariants of Poisson groupoids. In fact, a Poisson groupoid has associated a Lie bialgebroid and conversely, a Lie bialgebroid structure on the Lie algebroid of a (suitably simply connected) Lie groupoid can be integrated to give a Poisson groupoid structure on the underlying groupoid (see [MX1, MX2]). A Lie bialgebroid is a pair $\left(A, A^{*}\right)$ such that $A$ is a Lie algebroid and the dual vector bundle $A^{*}$ also carries a Lie algebroid structure which is compatible in a certain way with that on $A$. If $M$ is a Poisson manifold and on $T^{*} M$ (respectively, $T M$ ) we consider the cotangent Lie algebroid structure induced from the Poisson structure (respectively, the

2000 Mathematics Subject Classification: Primary 53D10, 53D17; Secondary 17B62.

Research partially supported by DGICYT grant PB97-1487 (Spain). DI wishes to thank Spanish Ministerio de Educación y Cultura for a FPU grant and University of La Laguna.

The paper is in final form and no version of it will be published elsewhere. 
trivial Lie algebroid structure) then the pair $\left(T M, T^{*} M\right)$ is a Lie bialgebroid (see [MX1]). Other interesting examples of Lie bialgebroids are Lie bialgebras [D], or equivalently, Lie bialgebroids over a single point.

On the other hand, a Jacobi structure on a manifold $M$ is a 2 -vector $\Lambda$ and a vector field $E$ on $M$ such that $[\Lambda, \Lambda]=2 E \wedge \Lambda$ and $[E, \Lambda]=0$, where [, ] is the Schouten-Nijenhuis bracket [L2]. If $(M, \Lambda, E)$ is a Jacobi manifold one can define a bracket of functions, the Jacobi bracket, in such a way that the space $C^{\infty}(M, \mathbb{R})$ endowed with the Jacobi bracket is a local Lie algebra in the sense of Kirillov [Ki]. Conversely, a local Lie algebra structure on $C^{\infty}(M, \mathbb{R})$ induces a Jacobi structure on $M[\mathrm{GL}, \mathrm{Ki}]$. Jacobi manifolds are natural generalizations of Poisson and contact manifolds. The 1-jet bundle $T^{*} M \times \mathbb{R} \rightarrow M$ associated with a Jacobi manifold $M$ admits a Lie algebroid structure [KS]. However, if on the vector bundle $T M \times \mathbb{R} \rightarrow M$ we consider the natural Lie algebroid structure then the pair $\left(T M \times \mathbb{R}, T^{*} M \times \mathbb{R}\right)$ is not a Lie bialgebroid [IM2, V3]. This fact and some examples of linear Jacobi structures on vector bundles obtained in [IM1] motivated the introduction, in [IM2], of the definition of a generalized Lie bialgebroid, a generalization of the notion of a Lie bialgebroid. A generalized Lie bialgebroid is a pair $\left(\left(A, \phi_{0}\right),\left(A^{*}, X_{0}\right)\right)$, where $A$ is a Lie algebroid, $\phi_{0}$ is a 1-cocycle in the Lie algebroid cohomology complex of $A$ with trivial coefficients, $A^{*}$ is the dual bundle to $A$ which admits a Lie algebroid structure and $X_{0}$ is a 1-cocycle of $A^{*}$. Moreover, the Lie algebroids $A$ and $A^{*}$ and the 1-cocycles $\phi_{0}$ and $X_{0}$ must satisfy some compatibility conditions. When the 1-cocycles $\phi_{0}$ and $X_{0}$ vanish, we recover the notion of a Lie bialgebroid. Furthermore, it was proved that the base space $M$ of a generalized Lie bialgebroid is a Jacobi manifold (see [IM2]).

Examples of generalized Lie bialgebroids are the so-called triangular generalized Lie bialgebroids. In fact, if $A$ is a Lie algebroid over $M$ with Schouten bracket $\llbracket, \rrbracket, \phi_{0}$ is a 1-cocycle and $P \in \Gamma\left(\wedge^{2} A\right)$ is a section of $\wedge^{2} A \rightarrow M$ such that $\llbracket P, P \rrbracket=2 i\left(\phi_{0}\right) P \wedge P$ then it was proved in [IM2] that the dual bundle $A^{*}$ admits a Lie algebroid structure in such a way that the pair $\left(\left(A, \phi_{0}\right),\left(A^{*}, X_{0}\right)\right)$ is a generalized Lie bialgebroid, where $X_{0}=-i\left(\phi_{0}\right) P$. Under the above conditions, we say that the triple $\left(A, \phi_{0}, P\right)$ is a triangular generalized Lie bialgebroid. An example of this situation are triangular Lie bialgebroids in the sense of Mackenzie and Xu [MX1] (in this case $\phi_{0}=0$ ). Other interesting examples are triangular generalized Lie bialgebras [IM2, IM3] and the triples of the form $\left(A=T M \times \mathbb{R}, \phi_{0}, P\right)$, where $(M, \Lambda, E)$ is a Jacobi manifold, $\phi_{0}=(0,1) \in \Omega^{1}(M) \oplus C^{\infty}(M, \mathbb{R}) \cong \Gamma\left(T^{*} M \times \mathbb{R}\right)$ and $P=(\Lambda, E) \in \mathcal{V}^{2}(M) \oplus \mathfrak{X}(M) \cong \Gamma\left(\wedge^{2}(T M \times \mathbb{R})\right)$ (see [IM2]).

In this paper, we consider two homology and cohomology theories associated with the dual Lie algebroid $A^{*}$ of a triangular generalized Lie bialgebroid $\left(A, \phi_{0}, P\right)$ and we prove that the vanishing of a certain cohomology class implies the existence of a duality between these homology and cohomology theories. In fact, if $\left(A, \phi_{0}, P\right)$ is a triangular generalized Lie bialgebroid over $M$ then the presence of the 1-cocycle $X_{0}=-i\left(\phi_{0}\right) P$ of $A^{*}$ allows us to deform the differential $d_{*}$ of the Lie algebroid $A^{*}$ and to define a new cohomology operator $d_{* X_{0}}$ given by $d_{* X_{0}}=d_{*}+e\left(X_{0}\right)$, where $e\left(X_{0}\right)$ denotes the exterior product by $X_{0}$. Thus, we have two cohomologies: the cohomology of the Lie algebroid $A^{*}, H^{*}\left(A^{*}\right)$, and the $X_{0^{-}}$-cohomology of $A^{*}, H_{X_{0}}^{*}\left(A^{*}\right)$. We show that the cohomology $H^{*}\left(A^{*}\right)$ (respectively, $H_{X_{0}}^{*}\left(A^{*}\right)$ ) is related with the cohomology of the local Lie algebra $C^{\infty}(M, \mathbb{R})$ relative to the representation of $C^{\infty}(M, \mathbb{R})$ on itself given by the hamiltonian 
vector fields (respectively, by the Jacobi bracket) of $M$. These representations also allow us to introduce two homology operators $\mathfrak{d}$ and $\mathfrak{d}_{X_{0}}$ on $\Gamma\left(\wedge^{*} A^{*}\right)=\oplus_{k} \Gamma\left(\wedge^{k} A^{*}\right)$. Moreover, if $\llbracket, \rrbracket_{*}$ is the Schouten bracket of $A^{*}$, we prove that $-\mathfrak{d}$ and $-\mathfrak{d}_{X_{0}}$ are generating operators of the Gerstenhaber algebra $\left(\Gamma\left(\wedge^{*} A^{*}\right), \wedge, \llbracket, \rrbracket *\right)$. Therefore, if $n$ is the rank of $A$ then, using the results of $[\mathrm{X}]$, we deduce that $-\mathfrak{d}$ and $-\mathfrak{d}_{X_{0}}$ define two flat $A^{*}$-connections $\nabla$ and $\nabla^{X_{0}}$ on $\wedge^{n} A^{*} \rightarrow M$ and the corresponding homologies $H_{*}\left(A^{*}, \nabla\right)$ and $H_{*}\left(A^{*}, \nabla^{X_{0}}\right)$.

On the other hand, if the vector bundle $A$ is orientable we introduce, in a natural way, the modular class $\mathcal{M}_{\left(A, \phi_{0}, P\right)}$ of the triple $\left(A, \phi_{0}, P\right)$ as an element of the first cohomology group $H^{1}\left(A^{*}\right)$ and we show that if $\mathcal{M}_{\left(A, \phi_{0}, P\right)}$ vanishes then

$$
H^{k}\left(A^{*}\right) \cong H_{n-k}\left(A^{*}, \nabla\right), \quad H_{X_{0}}^{k}\left(A^{*}\right) \cong H_{n-k}\left(A^{*}, \nabla^{X_{0}}\right), \text { for all } k .
$$

This theorem generalizes the results of $[\mathrm{BZ}, \mathrm{ELW}, \mathrm{X}]$ about the duality between the canonical homology and the Poisson cohomology for unimodular Poisson manifolds, the results of [Ko2] for unimodular triangular Lie bialgebroids and the results of [LLMP, V3] about the duality between the LJ-cohomology and the canonical LJ-homology (in the terminology of [LLMP]) for unimodular Jacobi manifolds. We also apply the above theorem to the particular case when $A$ is a real Lie algebra $\mathfrak{g}$ of finite dimension, $\phi_{0}=0$ and $P=r$ is a solution of the classical Yang-Baxter equation on $\mathfrak{g}$, that is, the pair $(\mathfrak{g}, r)$ is a triangular Lie bialgebra.

2. Lie algebroids. Homology and cohomology theories. If $M$ is a manifold of dimension $n$, we will denote by $C^{\infty}(M, \mathbb{R})$ the algebra of $C^{\infty}$ real-valued functions on $M$, by $\Omega^{k}(M)$ the space of $k$-forms, by $\mathcal{V}^{k}(M)$ the space of $k$-vectors, with $k \geq 2$, by $\mathfrak{X}(M)$ the Lie algebra of vector fields, by [, ] the Schouten-Nijenhuis bracket on $\mathcal{V}^{*}(M)=\oplus_{k} \mathcal{V}^{k}(M)$ and by $\delta$ the usual exterior differential on $\Omega^{*}(M)=\oplus_{k} \Omega^{k}(M)$.

A Lie algebroid $A$ over a manifold $M$ is a vector bundle $A$ over $M$ together with a Lie algebra structure $\llbracket, \rrbracket$ on the space $\Gamma(A)$ of the global cross sections of $A \rightarrow M$ and a bundle map $\rho: A \rightarrow T M$, called the anchor map, such that, if we also denote by $\rho: \Gamma(A) \rightarrow$ $\mathfrak{X}(M)$ the homomorphism of $C^{\infty}(M, \mathbb{R})$-modules induced by the anchor map, then:

(i) $\rho:(\Gamma(A), \llbracket, \rrbracket) \rightarrow(\mathfrak{X}(M),[]$,$) is a Lie algebra homomorphism and$

(ii) for all $f \in C^{\infty}(M, \mathbb{R})$ and for all $X, Y \in \Gamma(A)$, one has

$$
\llbracket X, f Y \rrbracket=f \llbracket X, Y \rrbracket+(\rho(X)(f)) Y .
$$

The triple $(A, \llbracket, \rrbracket, \rho)$ is called a Lie algebroid over $M$ (see $[\mathrm{M}, \mathrm{P}])$.

If $A$ is a Lie algebroid, the Lie bracket on the sections of $A$ can be extended to the so-called Schouten bracket $\llbracket$, $\rrbracket$ on the space $\Gamma\left(\wedge^{*} A\right)=\oplus_{k} \Gamma\left(\wedge^{k} A\right)$ of multi-sections of $A$ in such a way that

$$
\begin{aligned}
& \llbracket X, f \rrbracket=\rho(X)(f), \\
& \llbracket P, P^{\prime} \rrbracket=(-1)^{k k^{\prime}} \llbracket P^{\prime}, P \rrbracket, \\
& \llbracket P, P^{\prime} \wedge P^{\prime \prime} \rrbracket=\llbracket P, P^{\prime} \rrbracket \wedge P^{\prime \prime}+(-1)^{k^{\prime}(k+1)} P^{\prime} \wedge \llbracket P, P^{\prime \prime} \rrbracket \\
& (-1)^{k k^{\prime \prime}} \llbracket \llbracket P, P^{\prime} \rrbracket, P^{\prime \prime} \rrbracket+(-1)^{k^{\prime} k^{\prime \prime}} \llbracket \llbracket P^{\prime \prime}, P \rrbracket, P^{\prime} \rrbracket+(-1)^{k k^{\prime}} \llbracket \llbracket P^{\prime}, P^{\prime \prime} \rrbracket, P \rrbracket=0,
\end{aligned}
$$

for $f \in C^{\infty}(M, \mathbb{R}), X \in \Gamma(A), P \in \Gamma\left(\wedge^{k} A\right), P^{\prime} \in \Gamma\left(\wedge^{k^{\prime}} A\right)$ and $P^{\prime \prime} \in \Gamma\left(\wedge^{k^{\prime \prime}} A\right)$. The triple $\left(\Gamma\left(\wedge^{*} A\right)=\oplus_{k} \Gamma\left(\wedge^{k} A\right), \wedge, \mathbb{I}, \mathbb{\rrbracket}\right)$ is a Gerstenhaber algebra (see $\left.[\mathrm{M}]\right)$. 
REMARK 2.1. The definition of Schouten bracket considered here is the one given in [V2] (see also [BV, L1]). Some authors, see for example [ELW], define the Schouten bracket in another way. In fact, the relation between the Schouten bracket $\llbracket, \rrbracket^{\prime}$ in the sense of [ELW] and the Schouten bracket 【, \ in the sense of [V2] is the following $\llbracket P, Q \rrbracket^{\prime}=(-1)^{k+1} \llbracket P, Q \rrbracket$, for all $P \in \Gamma\left(\wedge^{k} A\right)$ and $Q \in \Gamma\left(\wedge^{*} A\right)$.

EXAMPLES 2.2. i) A trivial example of a Lie algebroid is the triple $(T M,[],, I d)$, where $M$ is a differentiable manifold and $I d: T M \rightarrow T M$ is the identity map.

ii) The Lie algebroid $(T M \times \mathbb{R},[],, \pi)$ : Let $M$ be a differentiable manifold and $A \rightarrow M$ a vector bundle over $M$. It is clear that $A \times \mathbb{R}$ is the total space of a vector bundle over $M$. Moreover, the dual bundle to $A \times \mathbb{R}$ is $A^{*} \times \mathbb{R}$ and the spaces $\Gamma\left(\wedge^{r}(A \times \mathbb{R})\right)$ and $\Gamma\left(\wedge^{k}\left(A^{*} \times \mathbb{R}\right)\right)$ can be identified with $\Gamma\left(\wedge^{r} A\right) \oplus \Gamma\left(\wedge^{r-1} A\right)$ and $\Gamma\left(\wedge^{k} A^{*}\right) \oplus \Gamma\left(\wedge^{k-1} A^{*}\right)$ in such a way that

$$
\begin{aligned}
& (P, Q)\left(\left(\alpha_{1}, f_{1}\right), \ldots,\left(\alpha_{r}, f_{r}\right)\right)=P\left(\alpha_{1}, \ldots, \alpha_{r}\right)+\sum_{i=1}^{r}(-1)^{i+1} f_{i} Q\left(\alpha_{1}, \ldots, \hat{\alpha}_{i}, \ldots, \alpha_{r}\right), \\
& (\alpha, \beta)\left(\left(X_{1}, g_{1}\right), \ldots,\left(X_{k}, g_{k}\right)\right)=\alpha\left(X_{1}, \ldots, X_{k}\right)+\sum_{i=1}^{k}(-1)^{i+1} g_{i} \beta\left(X_{1}, \ldots, \hat{X}_{i}, \ldots, X_{k}\right),
\end{aligned}
$$

for $(P, Q) \in \Gamma\left(\wedge^{r} A\right) \oplus \Gamma\left(\wedge^{r-1} A\right),(\alpha, \beta) \in \Gamma\left(\wedge^{k} A^{*}\right) \oplus \Gamma\left(\wedge^{k-1} A^{*}\right),\left(\alpha_{i}, f_{i}\right) \in \Gamma\left(A^{*}\right) \oplus$ $C^{\infty}(M, \mathbb{R})$ and $\left(X_{j}, g_{j}\right) \in \Gamma(A) \oplus C^{\infty}(M, \mathbb{R})$, with $i \in\{1, \ldots, r\}$ and $j \in\{1, \ldots, k\}$.

Under these identifications, the contractions and the exterior products are given by

$$
\begin{array}{lll}
i(\alpha, \beta)(P, Q) & =\left(i(\alpha) P+i(\beta) Q,(-1)^{k} i(\alpha) Q\right), & \text { if } k \leq r, \\
i(\alpha, \beta)(P, Q) & =0, & \text { if } k>r, \\
i(P, Q)(\alpha, \beta) & =\left(i(P) \alpha+i(Q) \beta,(-1)^{r} i(P) \beta\right), & \text { if } r \leq k, \\
i(P, Q)(\alpha, \beta) & =0, & \text { if } r>k, \\
(P, Q) \wedge\left(P^{\prime}, Q^{\prime}\right) & =\left(P \wedge P^{\prime}, Q \wedge P^{\prime}+(-1)^{r} P \wedge Q^{\prime}\right), & \\
(\alpha, \beta) \wedge\left(\alpha^{\prime}, \beta^{\prime}\right) & =\left(\alpha \wedge \alpha^{\prime}, \beta \wedge \alpha^{\prime}+(-1)^{k} \alpha \wedge \beta^{\prime}\right), &
\end{array}
$$

for $\left(P^{\prime}, Q^{\prime}\right) \in \Gamma\left(\wedge^{r^{\prime}} A\right) \oplus \Gamma\left(\wedge^{r^{\prime}-1} A\right)$ and $\left(\alpha^{\prime}, \beta^{\prime}\right) \in \Gamma\left(\wedge^{k^{\prime}} A^{*}\right) \oplus \Gamma\left(\wedge^{k^{\prime}-1} A^{*}\right)$.

Now, suppose that $A$ is the tangent bundle $T M$ of an arbitrary manifold $M$. In this case, the spaces $\Gamma\left(\wedge^{r}(T M \times \mathbb{R})\right)$ and $\Gamma\left(\wedge^{k}\left(T^{*} M \times \mathbb{R}\right)\right)$ can be identified with $\mathcal{V}^{r}(M) \oplus$ $\mathcal{V}^{r-1}(M)$ and $\Omega^{k}(M) \oplus \Omega^{k-1}(M)$.

Using the above identifications, we will exhibit a natural Lie algebroid structure on the vector bundle $T M \times \mathbb{R}$. If $\pi: T M \times \mathbb{R} \rightarrow T M$ is the canonical projection on the first factor and [, ] is the bracket given by

$$
[(X, f),(Y, g)]=([X, Y], X(g)-Y(f)),
$$

for $(X, f),(Y, g) \in \mathfrak{X}(M) \oplus C^{\infty}(M, \mathbb{R}) \cong \Gamma(T M \times \mathbb{R})$, then $(T M \times \mathbb{R},[],, \pi)$ is a Lie algebroid over $M$ (see [M, N]).

iii) The Lie algebroid $\left(T^{*} M \times \mathbb{R}, \llbracket, \rrbracket_{(\Lambda, E)}, \widetilde{\#}_{(\Lambda, E)}\right)$ of a Jacobi manifold: A Jacobi structure on a manifold $M$ is a 2-vector $\Lambda$ and a vector field $E$ on $M$ satisfying (see [L2])

$$
[\Lambda, \Lambda]=2 E \wedge \Lambda, \quad[E, \Lambda]=0
$$

The manifold $M$ endowed with a Jacobi structure is called a Jacobi manifold. If $(M, \Lambda, E)$ is a Jacobi manifold, we can define a bracket of functions (the Jacobi bracket) by the 
formula

$$
\{f, g\}=\Lambda(\delta f, \delta g)+f E(g)-g E(f)
$$

for all $f, g \in C^{\infty}(M, \mathbb{R})$. The space $C^{\infty}(M, \mathbb{R})$ endowed with this Jacobi bracket is a local Lie algebra in the sense of Kirillov (see [Ki]). Conversely, a structure of local Lie algebra on $C^{\infty}(M, \mathbb{R})$ defines a Jacobi structure on $M$ (see [GL, Ki]).

If $(M, \Lambda, E)$ is a Jacobi manifold, the 1 -jet bundle $T^{*} M \times \mathbb{R} \rightarrow M$ admits a Lie algebroid structure $\left(\llbracket, \rrbracket_{(\Lambda, E)}, \widetilde{\#}_{(\Lambda, E)}\right)$, where $\llbracket, \rrbracket_{(\Lambda, E)}$ and $\widetilde{\#}_{(\Lambda, E)}$ are defined by

$$
\begin{aligned}
& \llbracket(\alpha, f),(\beta, g) \rrbracket \\
&(\Lambda, E)=\left(\mathcal{L}_{\#_{\Lambda}(\alpha)} \beta-\mathcal{L}_{\#_{\Lambda}(\beta)} \alpha-\delta(\Lambda(\alpha, \beta))+f \mathcal{L}_{E} \beta-g \mathcal{L}_{E} \alpha-i(E)(\alpha \wedge \beta),\right. \\
&\left.\Lambda(\beta, \alpha)+\#_{\Lambda}(\alpha)(g)-\#_{\Lambda}(\beta)(f)+f E(g)-g E(f)\right), \\
& \widetilde{\#}_{(\Lambda, E)}(\alpha, f)= \#_{\Lambda}(\alpha)+f E,
\end{aligned}
$$

for $(\alpha, f),(\beta, g) \in \Omega^{1}(M) \oplus C^{\infty}(M, \mathbb{R})$ (see $\left.[\mathrm{KS}]\right)$. Here, $\mathcal{L}$ denotes the Lie derivative operator and $\#_{\Lambda}: \Omega^{1}(M) \rightarrow \mathfrak{X}(M)$ the homomorphism of $C^{\infty}(M, \mathbb{R})$-modules given by $\#_{\Lambda}(\alpha)=i(\alpha) \Lambda$. Note that from (4) and (5) it follows that

$$
\llbracket(\delta f, f),(\delta g, g) \rrbracket_{(\Lambda, E)}=(\delta\{f, g\},\{f, g\}) .
$$

In the particular case when $(M, \Lambda)$ is a Poisson manifold (i.e., $E=0$ ) we recover, by projection, the Lie algebroid $\left(T^{*} M, \llbracket, \rrbracket_{\Lambda}, \#_{\Lambda}\right)$, where $\llbracket, \rrbracket_{\Lambda}$ is the bracket of 1 -forms defined by (see $[\mathrm{F}])$ :

$$
\llbracket, \rrbracket_{\Lambda}: \Omega^{1}(M) \times \Omega^{1}(M) \rightarrow \Omega^{1}(M), \quad \llbracket \alpha, \beta \rrbracket_{\Lambda}=\mathcal{L}_{\#_{\Lambda}(\alpha)} \beta-\mathcal{L}_{\#_{\Lambda}(\beta)} \alpha-\delta(\Lambda(\alpha, \beta)) .
$$

Next, we will recall the definition of the Lie algebroid cohomology complex with trivial coefficients. For this purpose, we will recall the definition of the cohomology of a Lie algebra $\mathcal{A}$ with coefficients in an $\mathcal{A}$-module (we will follow [V2]).

Let $(\mathcal{A},[]$,$) be a real Lie algebra (not necessarily finite dimensional) and \mathcal{M}$ a real vector space endowed with a $\mathbb{R}$-bilinear multiplication

$$
\mathcal{A} \times \mathcal{M} \rightarrow \mathcal{M}, \quad(a, m) \mapsto a \cdot m
$$

such that

$$
\left[a_{1}, a_{2}\right] \cdot m=a_{1} \cdot\left(a_{2} \cdot m\right)-a_{2} \cdot\left(a_{1} \cdot m\right),
$$

for $a_{1}, a_{2} \in \mathcal{A}$ and $m \in \mathcal{M}$. In other words, we have a representation of $\mathcal{A}$ on $\mathcal{M}$. In such a case, a $k$-linear skew-symmetric mapping $c^{k}: \mathcal{A}^{k} \rightarrow \mathcal{M}$ is called an $\mathcal{M}$-valued $k$-cochain. These cochains form a real vector space $C^{k}(\mathcal{A} ; \mathcal{M})$ and the linear operator $\sigma^{k}: C^{k}(\mathcal{A} ; \mathcal{M}) \rightarrow C^{k+1}(\mathcal{A} ; \mathcal{M})$ given by

$$
\begin{aligned}
\left(\sigma^{k} c^{k}\right)\left(a_{0}, \ldots, a_{k}\right)= & \sum_{i=0}^{k}(-1)^{i} a_{i} \cdot c^{k}\left(a_{0}, \ldots, \hat{a}_{i}, \ldots, a_{k}\right)+ \\
& \sum_{i<j}(-1)^{i+j} c^{k}\left(\left[a_{i}, a_{j}\right], a_{0}, \ldots, \hat{a}_{i}, \ldots, \hat{a}_{j}, \ldots, a_{k}\right)
\end{aligned}
$$

defines a cohomology operator. Hence, we have the corresponding cohomology spaces $H^{k}(\mathcal{A} ; \mathcal{M})$. This cohomology is called the cohomology of the Lie algebra $\mathcal{A}$ with coefficients in $\mathcal{M}$, or relative to the given representation of $\mathcal{A}$ on $\mathcal{M}$. 
Now, if $(A, \llbracket, \rrbracket, \rho)$ is a Lie algebroid, we can define the representation of the Lie algebra $(\Gamma(A), \llbracket, \rrbracket)$ on the space $C^{\infty}(M, \mathbb{R})$ by $X \cdot f=\rho(X)(f)$, for $X \in \Gamma(A)$ and $f \in C^{\infty}(M, \mathbb{R})$. We will denote by $d$ the cohomology operator of the corresponding cohomology complex. Note that the space of the $k$-cochains which are $C^{\infty}(M, \mathbb{R})$-linear is just $\Gamma\left(\wedge^{k} A^{*}\right)$, where $A^{*}$ is the dual bundle to $A$. Moreover, we have that $d\left(\Gamma\left(\wedge^{k} A^{*}\right)\right) \subseteq \Gamma\left(\wedge^{k+1} A^{*}\right)$, for all $k$, and thus one can consider the subcomplex $\left(\Gamma\left(\wedge^{*} A^{*}\right), d_{\mid \Gamma\left(\wedge^{*} A^{*}\right)}\right)$. The cohomology of this subcomplex is the Lie algebroid cohomology with trivial coefficients and the restriction of $d$ to $\Gamma\left(\wedge^{*} A^{*}\right)$ is the differential of the Lie algebroid $A$ (see $[\mathrm{M}]$ ). We will denote the corresponding cohomology spaces by $H^{*}(A)$.

By the above definitions, a 1-cochain $\phi \in \Gamma\left(A^{*}\right)$ is a 1-cocycle if and only if

$$
\phi \llbracket X, Y \rrbracket=\rho(X)(\phi(Y))-\rho(Y)(\phi(X)), \text { for all } X, Y \in \Gamma(A) .
$$

On the other hand, if $X \in \Gamma(A)$ then one can introduce the Lie derivative by $X$ as the operator $\mathcal{L}_{X}: \Gamma\left(\wedge^{k} A^{*}\right) \rightarrow \Gamma\left(\wedge^{k} A^{*}\right)$ given by $\mathcal{L}_{X}=i(X) \circ d+d \circ i(X)$.

EXAMPLES 2.3. i) For the Lie algebroid $(T M,[],, I d)$, the Lie algebroid cohomology with trivial coefficients is just the de Rham cohomology of $M$.

ii) The Lie algebroid $(T M \times \mathbb{R},[],, \pi)$ : In this case, the dual bundle to $T M \times \mathbb{R}$ is $T^{*} M \times \mathbb{R}$ and, under the identification $\Gamma\left(\wedge^{k}\left(T^{*} M \times \mathbb{R}\right)\right) \cong \Omega^{k}(M) \oplus \Omega^{k-1}(M)$, the differential $\tilde{\delta}$ of the Lie algebroid $(T M \times \mathbb{R},[],, \pi)$ is given by

$$
\tilde{\delta}(\alpha, \beta)=(\delta \alpha,-\delta \beta),
$$

for $(\alpha, \beta) \in \Omega^{k}(M) \oplus \Omega^{k-1}(M)$.

iii) The Lie algebroid $\left(T^{*} M \times \mathbb{R}, \llbracket, \rrbracket_{(\Lambda, E)}, \widetilde{\#}_{(\Lambda, E)}\right)$ of a Jacobi manifold: If $\left(T^{*} M \times\right.$ $\left.\mathbb{R}, \llbracket, \rrbracket_{(\Lambda, E)}, \widetilde{\#}_{(\Lambda, E)}\right)$ is the Lie algebroid associated with a Jacobi manifold $(M, \Lambda, E)$ then, under the identification $\Gamma\left(\wedge^{k}(T M \times \mathbb{R})\right) \cong \mathcal{V}^{k}(M) \oplus \mathcal{V}^{k-1}(M)$, the differential $d^{(\Lambda, E)}$ of the Lie algebroid is given by (see [LLMP, LMP])

$$
d^{(\Lambda, E)}(P, Q)=(-[\Lambda, P]+k E \wedge P+\Lambda \wedge Q,[\Lambda, Q]-(k-1) E \wedge Q+[E, P]),
$$

for $(P, Q) \in \mathcal{V}^{k}(M) \oplus \mathcal{V}^{k-1}(M)$. The resultant cohomology $H_{L J}^{*}(M, \Lambda, E)$ was termed in [LMP] the Lichnerowicz-Jacobi cohomology (LJ-cohomology, for brevity) of $M$. This cohomology plays an important role in the geometric quantization of the Jacobi manifold $M$ and in the existence of prequantization representations for complex line bundles over $M$ (for more details, see [LLMP, LMP]).

Another description of the LJ-cohomology is the following. Consider the representation of the Lie algebra of functions on itself given by the hamiltonian vector fields, that is,

$$
\begin{aligned}
C^{\infty}(M, \mathbb{R}) \times C^{\infty}(M, \mathbb{R}) & \rightarrow C^{\infty}(M, \mathbb{R}) \\
(f, g) & \mapsto X_{f}(g)=\Lambda(\delta f, \delta g)+f E(g) .
\end{aligned}
$$

The resultant cohomology is called the H-Chevalley-Eilenberg cohomology of $M$ (see [LLMP, LMP]). The LJ-cohomology is just the cohomology of the subcomplex of the H-Chevalley-Eilenberg complex which consists of the 1-differentiable cochains, i.e., the $k$-linear skew-symmetric differential operators of order 1 (see [LLMP] for more details).

When $(M, \Lambda)$ is a Poisson manifold, the differential of the Lie algebroid $\left(T^{*} M, \llbracket, \rrbracket_{\Lambda}\right.$, $\left.\#_{\Lambda}\right)$ is the operator $d^{\Lambda}=-[\Lambda, \cdot]$. This operator was introduced by Lichnerowicz in [L1] to define the Poisson cohomology $H_{L P}^{*}(M, \Lambda)$ of $M$. 
Now, we will recall the definition of the homology operator associated with a Lie algebroid $A$ over $M$ of rank $n$ and a flat $A$-connection on $\wedge^{n} A \rightarrow M$ (see [X]).

Let $(A, \llbracket, \rrbracket, \rho)$ be a Lie algebroid over $M$ of rank $n$. An $A$-connection on a vector bundle $E \rightarrow M$ is a $\mathbb{R}$-bilinear mapping

$$
\begin{aligned}
\nabla: \Gamma(A) \times \Gamma(E) & \rightarrow \Gamma(E) \\
X \otimes s & \mapsto \nabla_{X} s
\end{aligned}
$$

such that

$$
\nabla_{f X} s=f \nabla_{X} s, \quad \nabla_{X}(f s)=(\rho(X)(f)) s+f \nabla_{X} s, \text { for all } f \in C^{\infty}(M, \mathbb{R}) .
$$

The curvature $R$ of an $A$-connection $\nabla$ may be defined as for the usual connections. $\nabla$ is said to be flat if $R$ vanishes.

Any $A$-connection on $\wedge^{n} A \rightarrow M$ defines a differential operator $D: \Gamma\left(\wedge^{k} A\right) \rightarrow$ $\Gamma\left(\wedge^{k-1} A\right)$ locally given by

$$
D(i(\omega) \Phi)=(-1)^{n-k}\left(i(d \omega) \Phi+\sum_{j=1}^{n} i\left(\alpha_{j} \wedge \omega\right) \nabla_{X_{j}} \Phi\right),
$$

where $\Phi \in \Gamma\left(\wedge^{n} A\right), \omega \in \Gamma\left(\wedge^{n-k} A^{*}\right),\left\{X_{j}\right\}$ is a local basis of $\Gamma(A)$ and $\left\{\alpha_{j}\right\}$ is the dual basis of $\Gamma\left(A^{*}\right)$. The operator $D$ generates the Gerstenhaber algebra $\left(\Gamma\left(\wedge^{*} A\right), \wedge, \llbracket, \rrbracket\right)$, that is, for all $U_{1} \in \Gamma\left(\wedge^{k_{1}} A\right)$ and $U_{2} \in \Gamma\left(\wedge^{k_{2}} A\right)$,

$$
\llbracket U_{1}, U_{2} \rrbracket=D\left(U_{1} \wedge U_{2}\right)-D U_{1} \wedge U_{2}-(-1)^{k_{1}} U_{1} \wedge D U_{2},
$$

or, equivalently

$$
\llbracket f, X \rrbracket=D(f X)-f D(X), \quad \llbracket X, Y \rrbracket=D(X \wedge Y)-(D X) Y+(D Y) X,
$$

for $f \in C^{\infty}(M, \mathbb{R})$ and $X, Y \in \Gamma(A)$.

Moreover, the connection $\nabla$ can be recovered from the operator $D$. More precisely, we have that

$$
\nabla_{X} \Phi=X \wedge D \Phi
$$

for all $X \in \Gamma(A)$ and $\Phi \in \Gamma\left(\wedge^{n} A\right)$.

In fact, (13) and (14) define a one-to-one correspondence between $A$-connections on $\wedge^{n} A \rightarrow M$ and linear operators $D$ generating the Gerstenhaber algebra $\left(\Gamma\left(\wedge^{*} A\right), \wedge, \llbracket, \mathbb{\rrbracket}\right)$. Under this correspondence, a flat $A$-connection $\nabla$ corresponds to an operator $D$ of zero square. Thus, a flat $A$-connection $\nabla$ induces a homology operator $\partial=-D$. The resultant homology $H_{*}(A, \nabla)$ is the homology of the Lie algebroid $A$ with respect to the flat $A$ connection $\nabla$.

If $\nabla$ and $\nabla^{\prime}$ are two $A$-connections on $\wedge^{n} A$, then there exists $\alpha \in \Gamma\left(A^{*}\right)$ such that

$$
\nabla_{X}^{\prime} \Phi=\nabla_{X} \Phi+\alpha(X) \Phi, \text { for } \Phi \in \Gamma\left(\wedge^{n} A\right) .
$$

As a consequence,

$$
D^{\prime}-D=i(\alpha)
$$

where $D$ and $D^{\prime}$ are their corresponding generating operators. Moreover, $\left(D^{\prime}\right)^{2}-D^{2}=$ $-i(d \alpha)$. Therefore, if $\nabla$ and $\nabla^{\prime}$ are two flat A-connections, then $\alpha \in \Gamma\left(A^{*}\right)$ is a 1-cocycle. In particular, if $\alpha=d f$, with $f \in C^{\infty}(M, \mathbb{R})$, one has that $H_{k}(A, \nabla) \cong H_{k}\left(A, \nabla^{\prime}\right)$, for all $k$ (for more details, see $[\mathrm{X}]$ ). 
REMARK 2.4. There are some differences of sign between the above formulas and those ones in $[\mathrm{X}]$. The reason is the definition of the Schouten bracket (see Remark 2.1).

ExAmple 2.5. Let $(M, \Lambda, E)$ be a Jacobi manifold of dimension $n$ and $\{$,$\} the as-$ sociated Jacobi bracket. Then, one can consider the flat $\left(T^{*} M \times \mathbb{R}\right)$-connection $\nabla$ on $\wedge^{n+1}\left(T^{*} M \times \mathbb{R}\right) \cong\{0\} \oplus \wedge^{n} T^{*} M$ defined by

$$
\nabla_{(\alpha, f)}(0, \Phi)=(0, f \delta i(E) \Phi+\alpha \wedge(\delta i(\Lambda) \Phi-n i(E) \Phi)),
$$

for $(\alpha, f) \in \Omega^{1}(M) \oplus C^{\infty}(M, \mathbb{R})$ and $\Phi \in \Omega^{n}(M)$. The connection $\nabla$ induces the homology operator $\partial^{(\Lambda, E)}: \Omega^{k}(M) \oplus \Omega^{k-1}(M) \rightarrow \Omega^{k-1}(M) \oplus \Omega^{k-2}(M)$ given by

$$
\begin{aligned}
\partial^{(\Lambda, E)}(\alpha, \beta)= & \left(i(\Lambda) \delta \alpha-\delta i(\Lambda) \alpha+k i(E) \alpha-\mathcal{L}_{E} \beta,\right. \\
& -i(\Lambda) \delta \beta+\delta i(\Lambda) \beta-(k-1) i(E) \beta+i(\Lambda) \alpha) .
\end{aligned}
$$

This homology operator was introduced by Vaisman in [V3]. The corresponding homology $H_{*}\left(T^{*} M \times \mathbb{R}, \nabla\right)$ was studied in [LLMP, V3].

If $(M, \Lambda)$ is an $n$-dimensional Poisson manifold, then the flat $T^{*} M$-connection on $\wedge^{n} T^{*} M$ defined by

$$
\nabla_{\alpha} \Phi=\alpha \wedge \delta i(\Lambda) \Phi, \text { for } \Phi \in \Omega^{n}(M) \text { and } \alpha \in \Omega^{1}(M),
$$

induces the homology operator $\partial^{\Lambda}$ given by the commutator of $i(\Lambda)$ and $\delta$, that is, $\partial^{\Lambda}=i(\Lambda) \delta-\delta i(\Lambda)$. The complex $\left(\Omega^{*}(M), \partial^{\Lambda}\right)$ is just the canonical homology complex introduced by Koszul $[\mathrm{K}]$ and studied by Brylinski in $[\mathrm{B}]$.

\section{Differential calculus on Lie algebroids in the presence of a 1-cocycle $\phi_{0}$}

3.1. $\phi_{0}$-cohomology. Let $(A, \llbracket, \rrbracket, \rho)$ be a Lie algebroid over $M$ and $\phi_{0} \in \Gamma\left(A^{*}\right)$ be a 1-cocycle in the Lie algebroid cohomology complex with trivial coefficients. Using (10), we can define a representation $\rho_{\phi_{0}}: \Gamma(A) \times C^{\infty}(M, \mathbb{R}) \rightarrow C^{\infty}(M, \mathbb{R})$ of the Lie algebra $(\Gamma(A), \llbracket, \rrbracket)$ on the space $C^{\infty}(M, \mathbb{R})$ given by

$$
\rho_{\phi_{0}}(X) f=\rho(X)(f)+\phi_{0}(X) f,
$$

for $X \in \Gamma(A)$ and $f \in C^{\infty}(M, \mathbb{R})$ (see [IM2]). Thus, one can consider the cohomology of the Lie algebra $(\Gamma(A), \llbracket, \rrbracket)$ with coefficients in $C^{\infty}(M, \mathbb{R})$ and the subcomplex $\Gamma\left(\wedge^{*} A^{*}\right)$ consisting of the $C^{\infty}(M, \mathbb{R})$-linear cochains. The cohomology operator $d_{\phi_{0}}: \Gamma\left(\wedge^{k} A^{*}\right) \rightarrow$ $\Gamma\left(\wedge^{k+1} A^{*}\right)$ of this subcomplex is called the $\phi_{0}$-differential of $A$. We have that

$$
d_{\phi_{0}} \alpha=d \alpha+\phi_{0} \wedge \alpha
$$

where $d$ is the differential of the Lie algebroid $(A, \llbracket, \rrbracket, \rho)$. The corresponding cohomology spaces will be denoted by $H_{\phi_{0}}^{*}(A)$ (see [IM2]).

REMARK 3.1. If $\phi_{0}$ is a 1-coboundary, that is, there exists $f \in C^{\infty}(M, \mathbb{R})$ such that $\phi_{0}=d f$ then the map $\Gamma\left(\wedge^{k} A^{*}\right) \rightarrow \Gamma\left(\wedge^{k} A^{*}\right), \phi \mapsto e^{-f} \phi$, induces an isomorphism between the cohomology groups $H^{k}(A)$ and $H_{\phi_{0}}^{k}(A)$.

EXAMPLES 3.2. i) If $\omega$ is a closed 1-form on a manifold $M$ then $\omega$ is a 1-cocycle for the trivial Lie algebroid $(T M,[],, I d)$ and we can consider the operator $d_{\omega}$. Some results about the cohomology defined by $d_{\omega}$ were obtained in [GL, LLMP, V1]. These results 
were used in the study of locally conformal Kähler and locally conformal symplectic structures.

ii) For the Lie algebroid $(T M \times \mathbb{R},[],, \pi)$, the 1-cochain $\phi_{0}=(0,1) \in \Omega^{1}(M) \oplus$ $C^{\infty}(M, \mathbb{R}) \cong \Gamma\left(T^{*} M \times \mathbb{R}\right)$ is a 1 -cocycle (see (11)) and the $\phi_{0}$-differential is given by

$$
\tilde{\delta}_{(0,1)}(\alpha, \beta)=(\delta \alpha, \alpha-\delta \beta),
$$

for $(\alpha, \beta) \in \Omega^{k}(M) \oplus \Omega^{k-1}(M)$. Note that, in this case, $H_{(0,1)}^{*}(T M \times \mathbb{R}) \cong\{0\}$.

iii) Let $\left(T^{*} M \times \mathbb{R}, \llbracket, \rrbracket(\Lambda, E), \widetilde{\#}_{(\Lambda, E)}\right)$ be the Lie algebroid associated with a Jacobi manifold $(M, \Lambda, E)$. Denote by $d^{(\Lambda, E)}$ the differential of this Lie algebroid. From (3) and (12), it follows that $X_{0}=(-E, 0) \in \mathfrak{X}(M) \oplus C^{\infty}(M, \mathbb{R}) \cong \Gamma(T M \times \mathbb{R})$ is a 1-cocycle. Then, using (2), (12) and (19), we obtain the following expression for the $X_{0}$-differential $\left(d^{(\Lambda, E)}\right)_{X_{0}}=\left(d^{(\Lambda, E)}\right)_{(-E, 0)}$,

$$
\begin{aligned}
\left(d^{(\Lambda, E)}\right)_{(-E, 0)}(P, Q)= & (-[\Lambda, P]+(k-1) E \wedge P+\Lambda \wedge Q, \\
& {[\Lambda, Q]-(k-2) E \wedge Q+[E, P]), }
\end{aligned}
$$

for $(P, Q) \in \mathcal{V}^{k}(M) \oplus \mathcal{V}^{k-1}(M)$. Note that $\left(d^{(\Lambda, E)}\right)_{(-E, 0)}$ is just the cohomology operator of the 1-differentiable Chevalley-Eilenberg cohomology complex of $M$ (see [GL, L2]). We denote by $H_{1-\operatorname{diff}}^{*}(M, \Lambda, E)$ the cohomology of this complex. In fact, in [GL] the 1-differentiable Chevalley-Eilenberg complex is described as the subcomplex of the Chevalley-Eilenberg complex which consists of the 1-differentiable cochains. We recall that the Chevalley-Eilenberg complex is the one defined by the representation of the Lie algebra of functions on itself given by

$$
C^{\infty}(M, \mathbb{R}) \times C^{\infty}(M, \mathbb{R}) \rightarrow C^{\infty}(M, \mathbb{R}), \quad(f, g) \mapsto\{f, g\},
$$

for all $f, g \in C^{\infty}(M, \mathbb{R})$, where $\{$,$\} is the Jacobi bracket of M$.

3.2. $\phi_{0}$-Schouten bracket. If $(A, \llbracket, \rrbracket, \rho)$ is a Lie algebroid then, imitating the definition of the Schouten bracket of two multilinear first-order differential operators on the space of $C^{\infty}$ real-valued functions on a manifold $N$ (see [BV]), we introduced in [IM2] the $\phi_{0}$-Schouten bracket of a $k$-section $P$ and a $k^{\prime}$-section $P^{\prime}$ as the $\left(k+k^{\prime}-1\right)$-section given by

$$
\llbracket P, P^{\prime} \rrbracket_{\phi_{0}}=\llbracket P, P^{\prime} \rrbracket+(-1)^{k+1}(k-1) P \wedge\left(i\left(\phi_{0}\right) P^{\prime}\right)-\left(k^{\prime}-1\right)\left(i\left(\phi_{0}\right) P\right) \wedge P^{\prime},
$$

where $\llbracket, \rrbracket$ is the usual Schouten bracket of $A$. The $\phi_{0}$-Schouten bracket satisfies the following properties. For $f \in C^{\infty}(M, \mathbb{R}), X, Y \in \Gamma(A), P \in \Gamma\left(\wedge^{k} A\right), P^{\prime} \in \Gamma\left(\wedge^{k^{\prime}} A\right)$ and $P^{\prime \prime} \in \Gamma\left(\wedge^{k^{\prime \prime}} A\right)$

$$
\begin{aligned}
& \llbracket X, f \rrbracket_{\phi_{0}}=\rho_{\phi_{0}}(X)(f), \\
& \llbracket X, Y \rrbracket_{\phi_{0}}=\llbracket X, Y \rrbracket \\
& \llbracket P, P^{\prime} \rrbracket_{\phi_{0}}=(-1)^{k k^{\prime}} \llbracket P^{\prime}, P \rrbracket_{\phi_{0}}, \\
& \llbracket P, P^{\prime} \wedge P^{\prime \prime} \rrbracket_{\phi_{0}}=\llbracket P, P^{\prime} \rrbracket_{\phi_{0}} \wedge P^{\prime \prime}+(-1)^{k^{\prime}(k+1)} P^{\prime} \wedge \llbracket P, P^{\prime \prime} \rrbracket_{\phi_{0}}-\left(i\left(\phi_{0}\right) P\right) \wedge P^{\prime} \wedge P^{\prime \prime}, \\
& (-1)^{k k^{\prime \prime}} \llbracket \llbracket P, P^{\prime} \rrbracket_{\phi_{0}}, P^{\prime \prime} \rrbracket_{\phi_{0}}+(-1)^{k^{\prime} k^{\prime \prime}} \llbracket \llbracket P^{\prime \prime}, P \rrbracket_{\phi_{0}}, P^{\prime} \rrbracket_{\phi_{0}}+(-1)^{k k^{\prime}} \llbracket \llbracket P^{\prime}, P^{\prime \prime} \rrbracket_{\phi_{0}}, P \rrbracket_{\phi_{0}}=0 .
\end{aligned}
$$

EXAmple 3.3. For the Lie algebroid $(T M \times \mathbb{R},[],, \pi)$, we have that the Schouten bracket is given by

$$
\left[(P, Q),\left(P^{\prime}, Q^{\prime}\right)\right]=\left(\left[P, P^{\prime}\right],(-1)^{k+1}\left[P, Q^{\prime}\right]-\left[Q, P^{\prime}\right]\right)
$$


for $(P, Q) \in \mathcal{V}^{k}(M) \oplus \mathcal{V}^{k-1}(M)$ and $\left(P^{\prime}, Q^{\prime}\right) \in \mathcal{V}^{k^{\prime}}(M) \oplus \mathcal{V}^{k^{\prime}-1}(M)$. Consequently (see (2) and (22)),

$$
\begin{aligned}
{\left[(P, Q),\left(P^{\prime}, Q^{\prime}\right)\right]_{(0,1)}=} & \left(\left[P, P^{\prime}\right]+(-1)^{k+1}(k-1) P \wedge Q^{\prime}-\left(k^{\prime}-1\right) Q \wedge P^{\prime},\right. \\
& \left.(-1)^{k+1}\left[P, Q^{\prime}\right]-\left[Q, P^{\prime}\right]+(-1)^{k+1}\left(k-k^{\prime}\right) Q \wedge Q^{\prime}\right) .
\end{aligned}
$$

Note that $(\Lambda, E) \in \Gamma\left(\wedge^{2}(T M \times \mathbb{R})\right)$ defines a Jacobi structure on $M$ if and only if $[(\Lambda, E),(\Lambda, E)]_{(0,1)}=0$ (see (3) and (23)). Moreover, using (21) and (23), we have that the $X_{0}$-differential $\left(d^{(\Lambda, E)}\right)_{X_{0}}=\left(d^{(\Lambda, E)}\right)_{(-E, 0)}$ of the Lie algebroid associated with a Jacobi manifold $(M, \Lambda, E)$ is given by

$$
\left(d^{(\Lambda, E)}\right)_{(-E, 0)}(P, Q)=-[(\Lambda, E),(P, Q)]_{(0,1)},
$$

for $(P, Q) \in \mathcal{V}^{k}(M) \oplus \mathcal{V}^{k-1}(M)$. Compare equation (24) with the expression of the differential of the Lie algebroid associated with a Poisson manifold (see iii) of Example 2.3).

4. Homology and cohomology theories and triangular generalized Lie bialgebroids. Let $(A, \llbracket, \rrbracket, \rho)$ be a Lie algebroid over $M$ and $\phi_{0} \in \Gamma\left(A^{*}\right)$ a 1-cocycle. Moreover, let $P \in \Gamma\left(\wedge^{2} A\right)$ be a bisection satisfying

$$
\llbracket P, P \rrbracket_{\phi_{0}}=\llbracket P, P \rrbracket-2 P \wedge i\left(\phi_{0}\right) P=0 .
$$

The triple $\left(A, \phi_{0}, P\right)$ is a triangular generalized Lie bialgebroid in the sense of [IM2] (note that if $\phi_{0}=0$ then the pair $(A, P)$ is a triangular Lie bialgebroid [MX1]).

Denote by $\#_{P}: \Gamma\left(A^{*}\right) \rightarrow \Gamma(A)$ the homomorphism of $C^{\infty}(M, \mathbb{R})$-modules given by

$$
\#_{P}(\phi)=i(\phi) P \text {. }
$$

If $A^{*} \rightarrow M$ is the dual bundle to $A,\left(A^{*}, \llbracket, \rrbracket_{*}, \rho_{*}\right)$ is a Lie algebroid, where the bracket $\llbracket, \rrbracket_{*}: \Gamma\left(A^{*}\right) \times \Gamma\left(A^{*}\right) \rightarrow \Gamma\left(A^{*}\right)$ and the map $\rho_{*}: \Gamma\left(A^{*}\right) \rightarrow \mathfrak{X}(M)$ are defined by

$$
\begin{aligned}
\llbracket \phi, \psi \rrbracket_{*} & =i\left(\#_{P}(\phi)\right) d_{\phi_{0}} \psi-i\left(\#_{P}(\psi)\right) d_{\phi_{0}} \phi+d_{\phi_{0}}(P(\phi, \psi)), \\
\rho_{*} & =\rho \circ \#_{P},
\end{aligned}
$$

for $\phi, \psi \in \Gamma\left(A^{*}\right)$. Moreover, $X_{0}=-\#_{P}\left(\phi_{0}\right) \in \Gamma(A)$ is a 1-cocycle for the Lie algebroid $\left(A^{*}, \llbracket, \rrbracket_{*}, \rho_{*}\right)$ (see $\left.[\mathrm{IM} 2]\right)$. In what follows, we will say that $\left(A^{*}, \llbracket, \rrbracket_{*}, \rho_{*}\right)$ is the dual Lie algebroid associated with the triple $\left((A, \llbracket, \rrbracket, \rho), \phi_{0}, P\right)$.

ExAmple 4.1. Let $(M, \Lambda, E)$ be a Jacobi manifold. Then, $(0,1) \in \Omega^{1}(M) \oplus C^{\infty}(M, \mathbb{R})$ is a 1-cocycle in the cohomology of the algebroid $(T M \times \mathbb{R},[],, \pi)$ and the pair $(\Lambda, E) \in$ $\mathcal{V}^{2}(M) \oplus \mathfrak{X}(M) \cong \Gamma\left(\wedge^{2}(T M \times \mathbb{R})\right)$ satisfies $[(\Lambda, E),(\Lambda, E)]_{(0,1)}=0$ (see Example 3.3).

Thus, $((T M \times \mathbb{R},[],, \pi),(0,1),(\Lambda, E))$ is a triangular generalized Lie bialgebroid. In this case, the dual Lie algebroid is just the algebroid $\left(T^{*} M \times \mathbb{R}, \llbracket, \rrbracket_{(\Lambda, E)}, \widetilde{\#}_{(\Lambda, E)}\right)$ associated with the Jacobi structure. Indeed, using (2), (5), (20) and (26), we deduce

$$
\begin{aligned}
\llbracket(\alpha, f),(\beta, g) \rrbracket_{(\Lambda, E)}= & i\left(\#_{(\Lambda, E)}(\alpha, f)\right)\left(\tilde{\delta}_{(0,1)}(\beta, g)\right)-i\left(\#_{(\Lambda, E)}(\beta, g)\right)\left(\tilde{\delta}_{(0,1)}(\alpha, f)\right) \\
& +\tilde{\delta}_{(0,1)}((\Lambda, E)((\alpha, f),(\beta, g))), \\
\widetilde{\#}_{(\Lambda, E)}= & \pi \circ \#_{(\Lambda, E)},
\end{aligned}
$$

for $(\alpha, f),(\beta, g) \in \Omega^{1}(M) \oplus C^{\infty}(M, \mathbb{R})$. 
4.1. Cohomology theories and triangular generalized Lie bialgebroids. The following result shows an explicit expression of the differential $d_{*}$ and of the $X_{0}$-differential $d_{*} X_{0}$ of the dual Lie algebroid $\left(A^{*}, \llbracket, \rrbracket *, \rho_{*}\right)$.

Proposition 4.2. Let $\left((A, \llbracket, \rrbracket, \rho), \phi_{0}, P\right)$ be a triangular generalized Lie bialgebroid. Then,

$$
\begin{gathered}
d_{*} Q=-\llbracket P, Q \rrbracket+P \wedge i\left(\phi_{0}\right) Q-k X_{0} \wedge Q, \\
d_{* X_{0}} Q=-\llbracket P, Q \rrbracket_{\phi_{0}},
\end{gathered}
$$

for $Q \in \Gamma\left(\wedge^{k} A\right)$, where $d_{*}$ (respectively, $d_{* X_{0}}$ ) is the differential (respectively, the $X_{0^{-}}$ differential) of the dual Lie algebroid $\left(A^{*}, \llbracket, \rrbracket *, \rho_{*}\right)$.

Proof. Let $\phi, \psi$ two sections of $A^{*}$ and $X$ a section of $A$. A direct computation, using (18) and (27), proves that

$$
\rho_{* X_{0}}(\alpha)(f)=\rho_{\phi_{0}}\left(\#_{P}(\alpha)\right)(f),
$$

for $\alpha \in \Gamma\left(A^{*}\right)$ and $f \in C^{\infty}(M, \mathbb{R})$. Then, from (9), (27) and (28), we obtain that

$$
\begin{aligned}
d_{* X_{0}} X(\phi, \psi)= & \rho_{* X_{0}}(\phi)(\psi(X))-\rho_{* X_{0}}(\psi)(\phi(X))-\llbracket \phi, \psi \rrbracket_{*}(X) \\
= & \rho_{\phi_{0}}\left(\#_{P}(\phi)\right)(\psi(X))-\rho_{\phi_{0}}(\# P(\psi))(\phi(X)) \\
& -\left(i\left(\#_{P}(\phi)\right) d_{\phi_{0}} \psi-i\left(\#_{P}(\psi)\right) d_{\phi_{0}} \phi+d_{\phi_{0}}(P(\phi, \psi))\right)(X) \\
= & \rho_{\phi_{0}}(X)(P(\phi, \psi))+\psi\left(\llbracket \#_{P}(\phi), X \rrbracket\right)-\phi\left(\llbracket \# \#_{P}(\psi), X \rrbracket\right) .
\end{aligned}
$$

On the other hand, if $\mathcal{L}$ denotes the Lie derivative of the algebroid $A$, we have that

$$
\llbracket Z, i(\omega) Q \rrbracket=i(Q)\left(\mathcal{L}_{Z} \omega\right)+i(\omega) \llbracket Z, Q \rrbracket,
$$

for $\omega \in \Gamma\left(\wedge^{k} A^{*}\right), Q \in \Gamma\left(\wedge^{k} A\right)$ and $Z \in \Gamma(A)$. Using this fact and (1), we get that

$$
\begin{aligned}
\rho(X)(P(\phi, \psi)) & =\left(\mathcal{L}_{X} \psi\right)\left(\#_{P}(\phi)\right)-\psi\left(\llbracket \#_{P}(\phi), X \rrbracket\right) \\
& =-\left(\mathcal{L}_{X} \phi\right)\left(\#_{P}(\psi)\right)+\phi\left(\llbracket \#_{P}(\psi), X \rrbracket\right) .
\end{aligned}
$$

Substituting these expressions in (29), we deduce that

$$
d_{* X_{0}} X(\phi, \psi)=-\rho(X)(P(\phi, \psi))+\left(\mathcal{L}_{X} \psi\right)\left(\#_{P}(\phi)\right)-\left(\mathcal{L}_{X} \phi\right)\left(\#_{P}(\psi)\right)+\phi_{0}(X) P(\phi, \psi) .
$$

Finally, using (22) and the properties of the Schouten bracket, we conclude that

$$
d_{* X_{0}} X(\phi, \psi)=-\left(\llbracket P, X \rrbracket-\phi_{0}(X) P\right)(\phi, \psi)=-\llbracket P, X \rrbracket_{\phi_{0}}(\phi, \psi) .
$$

In order to prove that this relation holds for $Q \in \Gamma\left(\wedge^{k} A\right)$ it is sufficient to proceed by induction on $k$ and to use the following fact,

$$
d_{* X_{0}}\left(R \wedge R^{\prime}\right)=d_{* X_{0}} R \wedge R^{\prime}+(-1)^{r} R \wedge d_{* X_{0}} R^{\prime}-X_{0} \wedge R \wedge R^{\prime},
$$

for $R \in \Gamma\left(\wedge^{r} A\right)$ and $R^{\prime} \in \Gamma\left(\wedge^{*} A\right)$.

Finally, from (19), (22) and since $X_{0}=-i\left(\phi_{0}\right) P$, we deduce that

$$
d_{*} Q=-\llbracket P, Q \rrbracket+P \wedge i\left(\phi_{0}\right) Q-k X_{0} \wedge Q .
$$

Now, denote by $\#_{P}: A^{*} \rightarrow A$ the bundle map induced by the homomorphism of $C^{\infty}(M, \mathbb{R})$-modules $\#_{P}: \Gamma\left(A^{*}\right) \rightarrow \Gamma(A)$ and by $\#_{P}^{k}: \wedge^{k} A^{*} \rightarrow \wedge^{k} A$ the bundle maps characterized by

$$
\#_{P}^{k}\left(\phi_{1} \wedge \ldots \wedge \phi_{k}\right)=\#_{P}\left(\phi_{1}\right) \wedge \ldots \wedge \#_{P}\left(\phi_{k}\right), \quad \text { for } \phi_{1}, \ldots, \phi_{k} \in \Gamma\left(A^{*}\right) .
$$


Proposition 4.3. Let $\left((A, \llbracket, \rrbracket, \rho), \phi_{0}, P\right)$ be a triangular generalized Lie bialgebroid. Then:

i) The map $\#_{P}: A^{*} \rightarrow A$ defines a homomorphism between the Lie algebroids $\left(A^{*}\right.$, $\left.\llbracket, \rrbracket_{*}, \rho_{*}\right)$ and $(A, \llbracket, \rrbracket, \rho)$.

ii) For all $k, d_{*} \circ \#_{P}^{k-1}=-\#_{P}^{k} \circ d\left(\right.$ resp. $\left.\left(d_{*}\right)_{X_{0}} \circ \#_{P}^{k-1}=-\#_{P}^{k} \circ d_{\phi_{0}}\right)$.

iii) The map $\#_{P}^{k}: \Gamma\left(\wedge^{k} A^{*}\right) \rightarrow \Gamma\left(\wedge^{k} A\right)$ induces a homomorphism between the cohomology groups $H^{k}(A)$ and $H^{k}\left(A^{*}\right)$ (resp. $H_{\phi_{0}}^{k}(A)$ and $\left.H_{X_{0}}^{k}\left(A^{*}\right)\right)$.

Proof. Note that

$$
\#_{P}^{2}(\gamma)(\phi, \psi)=\gamma\left(\#_{P}(\phi), \#_{P}(\psi)\right),
$$

for $\gamma \in \Gamma\left(\wedge^{2} A^{*}\right)$ and $\phi, \psi \in \Gamma\left(A^{*}\right)$. Using (1), (26) and (31), we obtain

$$
\frac{1}{2} i(\alpha) \llbracket P, P \rrbracket=\#_{P}^{2}(d \alpha)-\llbracket \#_{P}(\alpha), P \rrbracket,
$$

for $\alpha \in \Gamma\left(A^{*}\right)$. Thus, from (25), (32) and Proposition 4.2, we deduce that

$$
d_{*}\left(\#_{P}(\alpha)\right)=-\#_{P}^{2}(d \alpha) .
$$

Therefore, since $\rho_{*}=\rho \circ \#_{P},(33)$ implies that $\#_{P} \llbracket \phi, \psi \rrbracket_{*}=\llbracket \#_{P}(\phi), \#_{P}(\psi) \rrbracket$, for $\phi, \psi \in$ $\Gamma\left(A^{*}\right)$. This proves $\left.i\right)$.

ii) follows from $i),(19)$ and (30). Finally, using $i$ ) and $i i)$, we obtain $i i i)$.

On the other hand, if $\left(A, \phi_{0}, P\right)$ is a triangular generalized Lie bialgebroid over $M$, one can define a Jacobi bracket on the base space $M$ as follows (see [IM2])

$$
\{f, g\}:=d_{\phi_{0}} f\left(d_{* X_{0}} g\right)=-d_{\phi_{0}} g\left(d_{* X_{0}} f\right)=P\left(d_{\phi_{0}} f, d_{\phi_{0}} g\right) \text {, for } f, g \in C^{\infty}(M, \mathbb{R}) .
$$

The Jacobi structure $(\Lambda, E)$ induced by this bracket $\{$,$\} is characterized by$

$$
\begin{aligned}
& \Lambda(\delta f, \delta g)=d f\left(d_{*} g\right)=P(d f, d g), \text { for } f, g \in C^{\infty}(M, \mathbb{R}), \\
& E=-\rho\left(X_{0}\right)=\rho_{*}\left(\phi_{0}\right),
\end{aligned}
$$

(see [IM2] for more details).

Now, if $(A, \llbracket, \rrbracket, \rho)$ is a Lie algebroid over $M$ and $\phi_{0} \in \Gamma\left(A^{*}\right)$ is a 1-cocycle, we can consider the homomorphism of $C^{\infty}(M, \mathbb{R})$-modules $\left(\rho, \phi_{0}\right): \Gamma(A) \rightarrow \mathfrak{X}(M) \oplus C^{\infty}(M, \mathbb{R})$ given by

$$
\left(\rho, \phi_{0}\right)(X)=\left(\rho(X), \phi_{0}(X)\right), \quad \text { for all } X \in \Gamma(A) .
$$

This homomorphism induces a Lie algebroid homomorphism, which we also denote by $\left(\rho, \phi_{0}\right)$, between the Lie algebroids $(A, \llbracket, \rrbracket, \rho)$ and $(T M \times \mathbb{R},[],, \pi)$. We will denote by $\left(\rho, \phi_{0}\right)^{*}: T^{*} M \times \mathbb{R} \rightarrow A^{*}$ the adjoint homomorphism of $\left(\rho, \phi_{0}\right)$.

Proposition 4.4. Let $\left((A, \mathbb{I}, \mathbb{\rrbracket}, \rho), \phi_{0}, P\right)$ be a triangular generalized Lie bialgebroid over $M$. Suppose that $(\Lambda, E)$ is the associated Jacobi structure over $M$. Then, the bundle map $\left(\rho, \phi_{0}\right)^{*}: T^{*} M \times \mathbb{R} \rightarrow A^{*}$ induces a Lie algebroid homomorphism between $\left(T^{*} M \times\right.$ $\left.\mathbb{R}, \llbracket, \rrbracket(\Lambda, E), \widetilde{\#}_{(\Lambda, E)}\right)$ and the dual Lie algebroid $\left(A^{*}, \mathbb{I}, \rrbracket_{*}, \rho_{*}\right)$.

Proof. If $(\alpha, f) \in \Omega^{1}(M) \oplus C^{\infty}(M, \mathbb{R})$ and $\operatorname{dim} M=n$ then for every point $x \in M$ there exists an open subset $U$ of $M, x \in U$, and there exist $h_{i}, g_{i} \in C^{\infty}(U, \mathbb{R}), i \in\{1, \ldots, n+1\}$, 
such that on $U$

$$
(\alpha, f)=\sum_{i=1}^{n+1} h_{i}\left(\delta g_{i}, g_{i}\right) .
$$

Thus, it is sufficient to prove that $\left(\rho_{*} \circ\left(\rho, \phi_{0}\right)^{*}\right)(\delta f, f)=\tilde{\#}_{(\Lambda, E)}(\delta f, f)$, for all $f \in$ $C^{\infty}(M, \mathbb{R})$.

Now, we have that

$$
\left(\rho, \phi_{0}\right)^{*}(\delta f, f)=d_{\phi_{0}} f .
$$

Using this fact, (5), (19) and (35) we conclude that

$$
\begin{aligned}
\rho_{*}\left(\left(\rho, \phi_{0}\right)^{*}(\delta f, f)\right)(g) & =\rho_{*}\left(d_{\phi_{0}} f\right)(g)=d f\left(d_{*} g\right)+f \rho_{*}\left(\phi_{0}\right)(g) \\
& =\Lambda(\delta f, \delta g)+f E(g)=\widetilde{\#}_{(\Lambda, E)}(\delta f, f)(g),
\end{aligned}
$$

for all $g \in C^{\infty}(M, \mathbb{R})$.

Next, we will show that $\left(\rho, \phi_{0}\right)^{*}$ induces a homomorphism of Lie algebras, i.e.,

$$
\left(\rho, \phi_{0}\right)^{*} \llbracket(\alpha, f),(\beta, g) \rrbracket_{(\Lambda, E)}=\llbracket\left(\rho, \phi_{0}\right)^{*}(\alpha, f),\left(\rho, \phi_{0}\right)^{*}(\beta, g) \rrbracket_{*} \cdot
$$

Again, it is sufficient to prove this equality for pairs of the form $(\delta f, f)$ and $(\delta g, g)$.

But, from (6), (27), (34) and (37), we obtain that

$$
\begin{aligned}
\left(\rho, \phi_{0}\right)^{*} \llbracket(\delta f, f),(\delta g, g) \rrbracket_{(\Lambda, E)} & =\left(\rho, \phi_{0}\right)^{*}(\delta\{f, g\},\{f, g\})=d_{\phi_{0}}\{f, g\} \\
& =d_{\phi_{0}}\left(P\left(d_{\phi_{0}} f, d_{\phi_{0}} g\right)\right)=\llbracket d_{\phi_{0}} f, d_{\phi_{0}} g \rrbracket_{*} \\
& =\llbracket\left(\rho, \phi_{0}\right)^{*}(\delta f, f),\left(\rho, \phi_{0}\right)^{*}(\delta g, g) \rrbracket_{*} .
\end{aligned}
$$

Using Proposition 4.4, we deduce that

Corollary 4.5. Let $\left((A, \llbracket, \rrbracket, \rho), \phi_{0}, P\right)$ be a triangular generalized Lie bialgebroid over $M$. Suppose that $(\Lambda, E)$ is the associated Jacobi structure on $M$ and that $\left(\rho, \phi_{0}\right)^{k}$ : $\Gamma\left(\wedge^{k} A\right) \rightarrow \Gamma\left(\wedge^{k}(T M \times \mathbb{R})\right)$ is the homomorphism of $C^{\infty}(M, \mathbb{R})$-modules induced by the $\operatorname{map}\left(\rho, \phi_{0}\right): \Gamma(A) \rightarrow \mathfrak{X}(M) \oplus C^{\infty}(M, \mathbb{R}) \cong \Gamma(T M \times \mathbb{R})$.

i) If $d^{(\Lambda, E)}$ is the cohomology operator of the LJ-cohomology complex of $M$ and $d_{*}$ is the differential of the dual Lie algebroid $\left(A^{*}, \llbracket, \rrbracket_{*}, \rho_{*}\right)$ then $d^{(\Lambda, E)} \circ\left(\rho, \phi_{0}\right)^{k}=$ $\left(\rho, \phi_{0}\right)^{k+1} \circ d_{*}$.

ii) If $H_{L J}^{*}(M, \Lambda, E)$ is the LJ-cohomology of $M$ then the map $\left(\rho, \phi_{0}\right)^{k}$ induces a homomorphism between the cohomology groups $H^{k}\left(A^{*}\right)$ and $H_{L J}^{k}(M, \Lambda, E)$.

Note that $\left(\rho, \phi_{0}\right)\left(X_{0}\right)=(-E, 0)$. Therefore, from (19), (21) and Corollary 4.5, we conclude that

Corollary 4.6. Under the same hypotheses as in Corollary 4.5, we have that:

i) For all $k,\left(d^{(\Lambda, E)}\right)_{(-E, 0)} \circ\left(\rho, \phi_{0}\right)^{k}=\left(\rho, \phi_{0}\right)^{k+1} \circ d_{* X_{0}}$, where $X_{0}=-\#_{P}\left(\phi_{0}\right)$.

ii) If $H_{1-d i f f}^{*}(M, \Lambda, E)$ is the 1-differentiable Chevalley-Eilenberg cohomology of $M$ then the map $\left(\rho, \phi_{0}\right)^{k}$ induces a homomorphism between the cohomology groups $H_{X_{0}}^{k}\left(A^{*}\right)$ and $H_{1-d i f f}^{k}(M, \Lambda, E)$.

REMARK 4.7. A triangular generalized Lie bialgebroid is an example of a generalized Lie bialgebroid (see [IM2]). A generalized Lie bialgebroid over a manifold $M$ is a pair $\left(\left((A, \llbracket, \rrbracket, \rho), \phi_{0}\right),\left(\left(A^{*}, \llbracket, \rrbracket \rrbracket_{*}, \rho_{*}\right), X_{0}\right)\right)$, where $(A, \llbracket, \rrbracket, \rho)$ is a Lie algebroid over $M, \phi_{0}$ is 
a 1-cocycle in the Lie algebroid cohomology complex of $A$ with trivial coefficients, $A^{*}$ is the dual bundle to $A$ which admits a Lie algebroid structure $\left(\llbracket, \rrbracket_{*}, \rho_{*}\right)$ and $X_{0}$ is a 1-cocycle of $A^{*}$. Moreover, the Lie algebroids $A$ and $A^{*}$ and the 1-cocycles $\phi_{0}$ and $X_{0}$ must satisfy the following conditions

$$
\begin{aligned}
& d_{* X_{0}} \llbracket X, Y \rrbracket=\llbracket X, d_{* X_{0}} Y \rrbracket_{\phi_{0}}-\llbracket Y, d_{* X_{0}} X \rrbracket_{\phi_{0}} \\
& \phi_{0}\left(X_{0}\right)=0, \quad \rho\left(X_{0}\right)=-\rho_{*}\left(\phi_{0}\right) \\
& i\left(\phi_{0}\right) d_{* X_{0}} X+d_{* X_{0}}\left(\phi_{0}(X)\right)+\llbracket X_{0}, X \rrbracket=0 .
\end{aligned}
$$

for $X, Y \in \Gamma(A)$ (see [IM2]). When $\phi_{0}=0$ and $X_{0}=0$, we recover the notion of a Lie bialgebroid.

Every generalized Lie bialgebroid on $M$ induces a Jacobi bracket $\{$,$\} on M .\{$,$\} is$ defined as in (34) and satisfies the following condition

$$
d_{\phi_{0}}\{f, g\}=\llbracket d_{\phi_{0}} f, d_{\phi_{0}} g \rrbracket_{*}, \text { for all } f, g \in C^{\infty}(M, \mathbb{R}) .
$$

Using these facts, one can prove that Proposition 4.4 and Corollaries 4.5 and 4.6 are true for generalized Lie bialgebroids (not necessarily triangular).

4.2. Homology theories and triangular generalized Lie bialgebroids. Let $\left(A, \phi_{0}, P\right)$ be a triangular generalized Lie bialgebroid over $M$. Then, it is possible to define a Lie algebroid structure $\left(\llbracket, \rrbracket_{*}, \rho_{*}\right)$ on the dual bundle $A^{*}$ and, moreover, $M$ admits a Jacobi structure $(\Lambda, E)$.

As we have previously mentioned, the LJ-cohomology (respectively, the 1-differentiable Chevalley-Eilenberg cohomology) of $M, H_{L J}^{*}(M, \Lambda, E)$, (respectively, $H_{1-\text { diff }}^{*}(M$, $\Lambda, E)$ ) is the cohomology of a subcomplex of the H-Chevalley-Eilenberg (respectively, the Chevalley-Eilenberg) complex of $M$. Furthermore, $H_{L J}^{*}(M, \Lambda, E)$ and $H_{1-\operatorname{diff}}^{*}(M, \Lambda, E)$ are related with the cohomologies $H^{*}\left(A^{*}\right)$ and $H_{X_{0}}^{*}\left(A^{*}\right)$ (see Corollaries 4.5 and 4.6).

Next, we will introduce two homologies on the dual Lie algebroid $\left(A^{*}, \llbracket, \rrbracket_{*}, \rho_{*}\right)$ associated with the triple $\left((A, \llbracket, \rrbracket, \rho), \phi_{0}, P\right)$. These homologies are related with the ones defined by the representations of the Lie algebra $\left(C^{\infty}(M, \mathbb{R}),\{\},\right)$ on itself using the hamiltonian vector fields and the Jacobi bracket $\{$,$\} of M$.

In order to describe these homologies, we recall the definition of the homology of a Lie algebra $\mathcal{A}$ with coefficients in an $\mathcal{A}$-module (see, for instance, [CE]).

Let $(\mathcal{A},[]$,$) be a real Lie algebra (not necessarily finite dimensional) and \mathcal{M}$ a real vector space endowed with a $\mathbb{R}$-bilinear multiplication $\mathcal{A} \times \mathcal{M} \rightarrow \mathcal{M},(a, m) \mapsto a \cdot m$ satisfying (8).

An $\mathcal{M}$-valued $k$-chain is an element of the vector space $C_{k}(\mathcal{A} ; \mathcal{M})=\mathcal{M} \otimes \wedge^{k} \mathcal{A}$. We can consider the linear operator $\delta_{k}: C_{k}(\mathcal{A} ; \mathcal{M}) \rightarrow C_{k-1}(\mathcal{A} ; \mathcal{M})$ characterized by

$$
\begin{aligned}
\delta_{k}\left(m \otimes\left(a_{1} \wedge \ldots \wedge a_{k}\right)\right)= & \sum_{i=1}^{k}(-1)^{i+1} a_{i} \cdot m \otimes\left(a_{1} \wedge \ldots \wedge \hat{a}_{i} \wedge \ldots \wedge a_{k}\right)+ \\
& \sum_{i<j}(-1)^{i+j} m \otimes\left(\left[a_{i}, a_{j}\right] \wedge a_{1} \wedge \ldots \wedge \hat{a}_{i} \wedge \ldots \wedge \hat{a}_{j} \wedge \ldots \wedge a_{k}\right),
\end{aligned}
$$

which satisfies $\delta_{k-1} \circ \delta_{k}=0$, for all $k$. Thus, we have the corresponding homology spaces $H_{k}(\mathcal{A} ; \mathcal{M})$. This homology is said to be the homology of the Lie algebra $\mathcal{A}$ with coefficients in $\mathcal{M}$ or relative to the given representation of $\mathcal{A}$ on $\mathcal{M}$. 
In particular, for a Jacobi manifold $(M, \Lambda, E)$ with associated Jacobi bracket $\{$, $\}$, we can consider the homology of the Lie algebra $\left(C^{\infty}(M, \mathbb{R}),\{\},\right)$ on itself relative to the representation defined by the hamiltonian vector fields (respectively, the Lie bracket $\{$,$\} ).$ This homology is called the H-Chevalley-Eilenberg (respectively, Chevalley-Eilenberg) homology associated with $M$.

Now, let $\left((A, \llbracket, \rrbracket, \rho), \phi_{0}, P\right)$ be a triangular generalized Lie bialgebroid over $M$. Denote by $(\Lambda, E)$ the associated Jacobi structure on $M$, by $C_{k}(M)$ the space of $k$-chains in the Chevalley-Eilenberg and H-Chevalley-Eilenberg homology complex of $M$ and by $\partial_{H C E}^{(\Lambda, E)}$ (respectively, $\partial_{C E}^{(\Lambda, E)}$ ) the homology operator of the H-Chevalley-Eilenberg (respectively, Chevalley-Eilenberg) complex.

Imitating the construction of the canonical homology operator of a Poisson manifold [B] and of the canonical LJ-homology operator of a Jacobi manifold [LLMP], we consider the skew-symmetric $k$-linear mapping $\tilde{\pi}_{k}: C^{\infty}(M, \mathbb{R}) \times\left({ }^{k} . \times C^{\infty}(M, \mathbb{R}) \rightarrow \Gamma\left(\wedge^{k} A^{*}\right)\right.$ defined by

$$
\tilde{\pi}_{k}\left(f_{1} \wedge \ldots \wedge f_{k}\right)=d_{\phi_{0}} f_{1} \wedge \ldots \wedge d_{\phi_{0}} f_{k} .
$$

This mapping induces a linear mapping $\pi_{k}: C_{k}(M) \rightarrow \Gamma\left(\wedge^{k} A^{*}\right)$ characterized by

$$
\pi_{k}\left(f \otimes\left(f_{1} \wedge \ldots \wedge f_{k}\right)\right)=f d_{\phi_{0}} f_{1} \wedge \ldots \wedge d_{\phi_{0}} f_{k} .
$$

A direct computation, using (19), shows that

$$
\begin{gathered}
d_{\phi_{0}}\left(d_{\phi_{0}} f_{1} \wedge \ldots \wedge d_{\phi_{0}} f_{k}\right)=-(k-1) \phi_{0} \wedge d_{\phi_{0}} f_{1} \wedge \ldots \wedge d_{\phi_{0}} f_{k}, \\
i(P)(\alpha \wedge \beta)=i\left(\#_{P}(\alpha)\right) \beta+\alpha \wedge i(P) \beta,
\end{gathered}
$$

for all $f_{1}, \ldots, f_{k} \in C^{\infty}(M, \mathbb{R}), \alpha \in \Gamma\left(A^{*}\right)$ and $\beta \in \Gamma\left(\wedge^{*} A^{*}\right)$.

These formulas allow us to deduce the following facts

$$
\mathfrak{d} \circ \pi_{k}=-\pi_{k-1} \circ \partial_{H C E}^{(\Lambda, E)}, \quad \mathfrak{d}_{X_{0}} \circ \pi_{k}=-\pi_{k-1} \circ \partial_{C E}^{(\Lambda, E)},
$$

where $\mathfrak{d}: \Gamma\left(\wedge^{k} A^{*}\right) \rightarrow\left(\wedge^{k-1} A^{*}\right)$ and $\mathfrak{d}_{X_{0}}: \Gamma\left(\wedge^{k} A^{*}\right) \rightarrow\left(\wedge^{k-1} A^{*}\right)$ are the operators defined by

$$
\begin{gathered}
\mathfrak{d}(\phi)=i(P) d \phi-d i(P) \phi-k i\left(X_{0}\right) \phi+\phi_{0} \wedge i(P) \phi, \\
\mathfrak{d}_{X_{0}}(\phi)=\mathfrak{d}(\phi)-i\left(X_{0}\right) \phi .
\end{gathered}
$$

The following result proves that $-\mathfrak{d}$ and $-\mathfrak{d}_{X_{0}}$ are generating operators of the Gerstenhaber algebra $\left(\Gamma\left(\wedge^{*} A^{*}\right), \wedge, \mathbb{\llbracket}, \rrbracket_{*}\right)$ with zero square.

THEOREM 4.8. Let $\left((A, \llbracket, \rrbracket, \rho), \phi_{0}, P\right)$ be a triangular generalized Lie bialgebroid over $M$. If $\mathfrak{d}$ and $\mathfrak{d}_{X_{0}}$ are the operators defined in (39) and (40), then $-\mathfrak{d}$ and $-\mathfrak{d}_{X_{0}}$ are generating operators of the Gerstenhaber algebra $\left(\Gamma\left(\wedge^{*} A^{*}\right), \wedge, \llbracket, \mathbb{1} *\right)$ and

$$
\mathfrak{d}^{2}=0, \quad \mathfrak{d}_{X_{0}}^{2}=0 .
$$

Proof. Using (1), (27) and (39), we obtain that

$$
\mathfrak{d}(f \phi)-f \mathfrak{d} \phi=-\rho_{*}(\phi)(f)=-\llbracket f, \phi \rrbracket_{*},
$$

for $f \in C^{\infty}(M, \mathbb{R})$ and $\phi \in \Gamma\left(A^{*}\right)$.

Furthermore, from (19), (27), (38), (39) and since that $X_{0}=-\#_{P}\left(\phi_{0}\right)$, it follows that

$$
\mathfrak{d}(\phi \wedge \psi)-(\mathfrak{d} \phi) \psi+(\mathfrak{d} \psi) \phi=-\llbracket \phi, \psi \rrbracket_{*},
$$


for $\phi, \psi \in \Gamma\left(A^{*}\right)$. Thus, $-\mathfrak{d}$ is a generating operator of the Gerstenhaber algebra $\left(\Gamma\left(\wedge^{*} A^{*}\right)\right.$, $\left.\wedge, \llbracket, \rrbracket_{*}\right)$. Consequently, using (40), the fact that $X_{0}$ is a 1-cocycle and the results of $[\mathrm{X}]$ (see Section 2), we have that $-\mathfrak{d}_{X_{0}}$ is also a generating operator of the Gerstenhaber algebra $\left(\Gamma\left(\wedge^{*} A^{*}\right), \wedge, \llbracket, \rrbracket *\right)$.

Next, we will show that $\mathfrak{d}^{2}=0$.

Denote by $\gamma$ the commutator of $i(P)$ and $d$, that is,

$$
\gamma=[i(P), d]=i(P) d-d i(P) .
$$

Then,

$$
\gamma^{2}=-i(P) i\left(X_{0}\right) d-d i(P) i\left(X_{0}\right)
$$

Indeed, it is clear that

$$
\gamma^{2}=i(P) d i(P) d-d i(P) i(P) d+d i(P) d i(P) .
$$

Moreover, since $X_{0}=-\#_{P}\left(\phi_{0}\right)$ and

$$
[[i(R), d], i(Q)]=i(\llbracket R, Q \rrbracket), \text { for } R, Q \in \Gamma\left(\wedge^{*} A\right),
$$

we deduce that

$$
\begin{aligned}
\gamma i(P)-i(P) \gamma & =[[i(P), d], i(P)]=i(\llbracket P, P \rrbracket) \\
& =i\left(\llbracket P, P \rrbracket_{\phi_{0}}\right)-2 i\left(X_{0}\right) i(P)=-2 i\left(X_{0}\right) i(P) .
\end{aligned}
$$

On the other hand, using (41), we have that

$$
\gamma i(P)-i(P) \gamma=2 i(P) d i(P)-d i(P) i(P)-i(P) i(P) d .
$$

Therefore, from (45) and (46), we conclude

$$
i(P) d i(P)=-i\left(X_{0}\right) i(P)+\frac{1}{2}(d i(P) i(P)+i(P) i(P) d) .
$$

Substituting (47) in (43), we obtain (42).

Now, using (38), (39), (41), (42) and the fact that $d \phi_{0}=0$, we deduce that

$$
\mathfrak{d}^{2}(\phi)=k\left(\left[\left[i\left(X_{0}\right), d\right], i(P)\right]\right)(\phi)-\phi_{0} \wedge\left(\gamma i(P)-i(P) \gamma+2 i\left(X_{0}\right) i(P)\right)(\phi),
$$

for $\phi \in \Gamma\left(\wedge^{k} A^{*}\right)$. Thus, from (44), (45), (48) and since $0=d_{*} X_{0}=\llbracket P, X_{0} \rrbracket$, it follows that $\mathfrak{d}^{2}=0$.

Finally, using that $\mathfrak{d}$ is a generating operator of the Gerstenhaber algebra $\left(\Gamma\left(\wedge^{*} A^{*}\right), \wedge\right.$, $\left.\llbracket, \rrbracket_{*}\right)$, that $\mathfrak{d}^{2}=0$ and that $X_{0}$ is a 1 -cocycle for the Lie algebroid $\left(A^{*}, \llbracket, \rrbracket *, \rho_{*}\right)$, we conclude that $\mathfrak{d}_{X_{0}}^{2}=0$.

If the rank of $A$ is $n$ then the generating operators $-\mathfrak{d}$ and $-\mathfrak{d}_{X_{0}}$ define the following flat $A^{*}$-connections on $\wedge^{n} A^{*} \rightarrow M($ see $(14)$ and (38))

$$
\begin{aligned}
\nabla_{\alpha} \Phi & =-\alpha \wedge \mathfrak{d} \Phi=\alpha \wedge\left(d i(P) \Phi+n i\left(X_{0}\right) \Phi-\phi_{0} \wedge i(P) \Phi\right) \\
& =\alpha \wedge\left(d i(P) \Phi+(n-1) i\left(X_{0}\right) \Phi\right), \\
\nabla_{\alpha}^{X_{0}} \Phi=- & -\alpha \wedge \mathfrak{d}_{X_{0}} \Phi=\alpha \wedge\left(d i(P) \Phi+(n-1) i\left(X_{0}\right) \Phi-\phi_{0} \wedge i(P) \Phi\right) \\
= & \alpha \wedge\left(d i(P) \Phi+n i\left(X_{0}\right) \Phi\right),
\end{aligned}
$$

for all $\alpha \in \Gamma\left(A^{*}\right)$ and $\Phi \in \Gamma\left(\wedge^{n} A^{*}\right)$. 
4.3. Triangular generalized Lie bialgebroids, duality and modular class. In this Section, we will introduce the modular class for a triangular generalized Lie bialgebroid. Moreover, we will study the role played by this cohomology class in the duality between the cohomology and homology theories introduced in Sections 4.1 and 4.2.

Assume that $(A, \llbracket, \rrbracket, \rho)$ is an orientable Lie algebroid of rank $n$ and let $\nu \in \Gamma\left(\wedge^{n} A^{*}\right)$ be a nowhere vanishing $n$-section. If $\phi_{0} \in \Gamma\left(A^{*}\right)$ is a 1-cocycle and $P \in \Gamma\left(\wedge^{2} A\right)$ is a bisection satisfying $\llbracket P, P \rrbracket_{\phi_{0}}=0$, we can consider the dual Lie algebroid $\left(A, \llbracket, \rrbracket_{*}, \rho_{*}\right)$ associated with the triple $\left((A, \llbracket, \rrbracket, \rho), \phi_{0}, P\right)$.

Define the $*$-operator from $\Gamma\left(\wedge^{k} A\right)$ to $\Gamma\left(\wedge^{n-k} A^{*}\right)$ as follows

$$
* Q=i(Q) \nu, \text { for } Q \in \Gamma\left(\wedge^{k} A\right) .
$$

Clearly, * is an isomorphism of $C^{\infty}(M, \mathbb{R})$-modules. Moreover, we have that

$$
\partial_{0}(* Q)=(-1)^{k+1} *\left(d_{*} Q\right), \quad\left(\partial_{0}\right)_{X_{0}}(* Q)=(-1)^{k+1} *\left(d_{* X_{0}} Q\right),
$$

where $d_{*}$ (respectively, $d_{* X_{0}}$ ) is the differential of the Lie algebroid $\left(A^{*}, \llbracket, \rrbracket_{*}, \rho_{*}\right)$ (respectively, the $X_{0}$-differential) and $\partial_{0}$ and $\left(\partial_{0}\right)_{X_{0}}$ are the homology operators associated with the flat $A^{*}$-connections $\nabla_{0}$ and $\nabla_{0}^{X_{0}}$ on $\wedge^{n} A^{*} \rightarrow M$ characterized by

$$
\left(\nabla_{0}\right)_{\alpha} \nu=0, \quad\left(\nabla_{0}^{X_{0}}\right)_{\alpha} \nu=\alpha\left(X_{0}\right) \nu
$$

for all $\alpha \in \Gamma\left(A^{*}\right)$. Thus, (51) implies that

$$
H^{k}\left(A^{*}\right) \cong H_{n-k}\left(A^{*}, \nabla_{0}\right), \quad H_{X_{0}}^{k}\left(A^{*}\right) \cong H_{n-k}\left(A^{*}, \nabla_{0}^{X_{0}}\right),
$$

for all $k \in\{0, \ldots, n\}$.

Now, we will compare the homology $H_{k}\left(A^{*}, \nabla_{0}\right)$ (respectively, $H_{k}\left(A^{*}, \nabla_{0}^{X_{0}}\right)$ ) with the homology of the dual Lie algebroid $\left(A^{*}, \llbracket, \rrbracket{ }_{*}, \rho_{*}\right)$ associated with the flat $A^{*}$-connection $\nabla$ (respectively, $\nabla^{X_{0}}$ ) defined in (49) (respectively, (50)).

In fact, using (49), (50) and (52), we obtain that

$$
\nabla_{\alpha} \nu-\left(\nabla_{0}\right)_{\alpha} \nu=\nabla_{\alpha}^{X_{0}} \nu-\left(\nabla_{0}^{X_{0}}\right)_{\alpha} \nu=\alpha \wedge\left(d i(P) \nu+(n-1) i\left(X_{0}\right) \nu\right),
$$

for all $\alpha \in \Gamma\left(A^{*}\right)$.

On the other hand, if $\mathcal{L}$ is the Lie derivative on $A$, from (38), we deduce that

$$
\begin{aligned}
0=d(i(P)(\alpha \wedge \nu)) & =d\left(i\left(\#_{P}(\alpha)\right) \nu\right)+d \alpha \wedge i(P) \nu-\alpha \wedge d i(P) \nu \\
& =\mathcal{L}_{\#_{P}(\alpha)} \nu+d \alpha \wedge i(P) \nu-\alpha \wedge d i(P) \nu \\
& =\mathcal{L}_{\#_{P}(\alpha)} \nu+i(P)(d \alpha) \nu-\alpha \wedge d i(P) \nu \\
0=i\left(X_{0}\right)(\alpha \wedge \nu) & =\alpha\left(X_{0}\right) \nu-\alpha \wedge i\left(X_{0}\right) \nu .
\end{aligned}
$$

Substituting in (54), we conclude that

$$
\nabla_{\alpha} \nu-\left(\nabla_{0}\right)_{\alpha} \nu=\nabla_{\alpha}^{X_{0}} \nu-\left(\nabla_{0}^{X_{0}}\right)_{\alpha} \nu=\mathcal{L}_{\#_{P}(\alpha)} \nu+\left(i(P)(d \alpha)+(n-1) \alpha\left(X_{0}\right)\right) \nu .
$$

Now, we consider the section $\mathcal{M}_{\left(A, \phi_{0}, P\right)}^{\nu} \in \Gamma(A)$ characterized by the condition

$$
\alpha\left(\mathcal{M}_{\left(A, \phi_{0}, P\right)}^{\nu}\right) \nu=\mathcal{L}_{\#_{P}(\alpha)} \nu+\left(i(P)(d \alpha)+(n-1) \alpha\left(X_{0}\right)\right) \nu
$$

for all $\alpha \in \Gamma\left(A^{*}\right)$. From (55) and (56), it follows that

$$
\nabla_{\alpha} \nu-\left(\nabla_{0}\right)_{\alpha} \nu=\nabla_{\alpha}^{X_{0}} \nu-\left(\nabla_{0}^{X_{0}}\right)_{\alpha} \nu=\alpha\left(\mathcal{M}_{\left(A, \phi_{0}, P\right)}^{\nu}\right) \nu,
$$


and, since $\nabla$ and $\nabla_{0}$ (respectively, $\nabla^{X_{0}}$ and $\nabla_{0}^{X_{0}}$ ) are flat $A^{*}$-connections, we have that $\mathcal{M}_{\left(A, \phi_{0}, P\right)}^{\nu}$ is a 1-cocycle for the Lie algebroid $\left(A^{*}, \llbracket, \rrbracket *, \rho_{*}\right)$ (see Section 2).

Furthermore, using (15), (16) and (57), we obtain that

$$
\mathfrak{d}-\partial_{0}=\mathfrak{d}_{X_{0}}-\left(\partial_{0}\right)_{X_{0}}=-i\left(\mathcal{M}_{\left(A, \phi_{0}, P\right)}^{\nu}\right),
$$

where $\mathfrak{d}$ (respectively, $\mathfrak{d}_{X_{0}}$ ) is the homology operator given by (39) (respectively, (40)).

The cohomology class $\mathcal{M}_{\left(A, \phi_{0}, P\right)}=\left[\mathcal{M}_{\left(A, \phi_{0}, P\right)}^{\nu}\right] \in H^{1}\left(A^{*}\right)$ does not depend on the chosen section $\nu$. In fact, if $\nu^{\prime} \in \Gamma\left(\wedge^{n} A^{*}\right)$ is another nowhere vanishing section, there exists $f \in C^{\infty}(M, \mathbb{R}), f \neq 0$ at every point, such that $\nu^{\prime}=f \nu$. We can suppose, without loss of generality, that $f>0$. Then, a direct computation, using (56), proves that

$$
\mathcal{M}_{\left(A, \phi_{0}, P\right)}^{\nu^{\prime}}=\mathcal{M}_{\left(A, \phi_{0}, P\right)}^{\nu}+d_{*}(\ln f) \text {. }
$$

Definition 4.9. The cohomology class $\mathcal{M}_{\left(A, \phi_{0}, P\right)}=\left[\mathcal{M}_{\left(A, \phi_{0}, P\right)}^{\nu}\right] \in H^{1}\left(A^{*}\right)$ is called the modular class of the triangular generalized Lie bialgebroid $\left(A, \phi_{0}, P\right)$. The triangular generalized Lie bialgebroid is said to be unimodular if its associated modular class $\mathcal{M}_{\left(A, \phi_{0}, P\right)}$ vanishes.

From (53) and (58), we conclude

THEOREM 4.10. Let $(A, \llbracket, \rrbracket, \rho)$ be an orientable Lie algebroid of rank $n, \phi_{0} \in \Gamma\left(A^{*}\right)$ a 1-cocycle and $P \in \Gamma\left(\wedge^{2} A\right)$ satisfying $\llbracket P, P \rrbracket_{\phi_{0}}=0$. If the triangular generalized bialgebroid $\left(A, \phi_{0}, P\right)$ is unimodular then

$$
H^{k}\left(A^{*}\right) \cong H_{n-k}\left(A^{*}, \nabla\right), \quad H_{X_{0}}^{k}\left(A^{*}\right) \cong H_{n-k}\left(A^{*}, \nabla^{X_{0}}\right),
$$

where $X_{0}=-\#_{P}\left(\phi_{0}\right)$, and $\nabla$ (respectively, $\nabla^{X_{0}}$ ) is the flat $A^{*}$-connection defined by (49) (respectively, (50)).

Next, we will relate the modular class of a triangular generalized Lie bialgebroid $\left(A, \phi_{0}, P\right)$ over $M$ with the modular class of the Lie algebroids $A$ and $A^{*}$ and with the modular class of the base Jacobi manifold $M$. For this purpose, we will recall the definition of the modular class of a Lie algebroid introduced by Evens, Lu and Weinsten [ELW] (see also $[\mathrm{W}]$ ) and the definition of the modular class of a Jacobi manifold introduced by Vaisman [V3] (see also [LLMP]).

Let $(M, \Lambda, E)$ be an orientable Jacobi manifold of dimension $m$ and $\Omega$ a volume form on $M$. We consider the section $\mathcal{M}_{(\Lambda, E)}^{\Omega}$ of the vector bundle $T M \times \mathbb{R} \rightarrow M$ given by

$$
\mathcal{M}_{(\Lambda, E)}^{\Omega}=\left(\mathcal{X}_{(\Lambda, E)}^{\Omega}-m E, \operatorname{div}_{\Omega} E\right) \in \mathfrak{X}(M) \oplus C^{\infty}(M, \mathbb{R}) \cong \Gamma(T M \times \mathbb{R}),
$$

where $\mathcal{X}_{(\Lambda, E)}^{\Omega}$ is the vector field characterized by the relation

$$
\mathcal{L}_{\#_{\Lambda}(\delta f)} \Omega=\left(\mathcal{X}_{(\Lambda, E)}^{\Omega}(f)\right) \Omega, \quad \text { for all } f \in C^{\infty}(M, \mathbb{R}),
$$

and $\operatorname{div}_{\Omega} E$ is the divergence of the vector field $E$ with respect to $\Omega$, that is, $\mathcal{L}_{E} \Omega=$ $\left(\operatorname{div}_{\Omega} E\right) \Omega$. The section $\mathcal{M}_{(\Lambda, E)}^{\Omega}$ is a 1-cocycle in the LJ-cohomology complex of $M$ and the cohomology class $\mathcal{M}_{(\Lambda, E)}=\left[\mathcal{M}_{(\Lambda, E)}^{\Omega}\right] \in H_{L J}^{1}(M, \Lambda, E)$ is the modular class of $M$ (see [LLMP, V3]). As we know (see Example 4.1), the triple $((T M \times \mathbb{R},[],, \pi),(0,1),(\Lambda, E))$ is a triangular generalized Lie bialgebroid and $\left(T^{*} M \times \mathbb{R}, \mathbb{\llbracket}, \mathbb{\rrbracket}(\Lambda, E), \tilde{\#}_{(\Lambda, E)}\right)$ is the dual Lie algebroid of $((T M \times \mathbb{R},[],, \pi),(0,1),(\Lambda, E))$. Using this fact, (2), (56), (59) and (60), we deduce that $\mathcal{M}_{(\Lambda, E)}$ is the modular class of $((T M \times \mathbb{R},[],, \pi),(0,1),(\Lambda, E))$. 
On the other hand, if $(\mathcal{E}, \llbracket, \rrbracket, \rho)$ is an orientable Lie algebroid of rank $n$ over an orientable manifold $M$ of dimension $m$, one can introduce the modular class of $\mathcal{E}$ as follows (see $[$ ELW] $)$. Let $\nu \in \Gamma\left(\wedge^{n} \mathcal{E}^{*}\right)$ be a nowhere vanishing $n$-section and let $\Omega \in \Omega^{m}(M)$ be a volume form on $M$. Then, we define the section $\mathcal{M}_{\mathcal{E}}^{(\nu, \Omega)}$ of $\mathcal{E}^{*} \rightarrow M$ given by

$$
\mathcal{M}_{\mathcal{E}}^{(\nu, \Omega)}(X)=\operatorname{div}_{\Omega} \rho(X)-\operatorname{div}_{\nu} X
$$

for all $X \in \Gamma(\mathcal{E})$, where $\operatorname{div}_{\Omega} \rho(X)$ is the divergence of the vector field $\rho(X)$ with respect to $\Omega$ and $\operatorname{div}_{\nu} X$ is the function on $M$ characterized by $\mathcal{L}_{X} \nu=\left(\operatorname{div}_{\nu} X\right) \nu$. The section $\mathcal{M}_{\mathcal{E}}^{(\nu, \Omega)}$ is a 1-cocycle in the Lie algebroid cohomology complex of $\mathcal{E}$ and the cohomology class $\mathcal{M}_{\mathcal{E}}=\left[\mathcal{M}_{\mathcal{E}}^{(\nu, \Omega)}\right] \in H^{1}(\mathcal{E})$ is the modular class of $\mathcal{E}$.

REMARK 4.11. Let $(M, \Lambda, E)$ be an orientable Jacobi manifold of dimension $m$ and $\left(T^{*} M \times \mathbb{R}, \llbracket, \rrbracket(\Lambda, E), \tilde{\#}_{(\Lambda, E)}\right)$ the 1 -jet Lie algebroid associated with $M$. Suppose that $\Omega \in \Omega^{m}(M)$ is a volume form on $M$ and denote by $V_{\Omega}$ the $m$-vector on $M$ defined by

$$
\alpha_{1} \wedge \ldots \wedge \alpha_{m}=V_{\Omega}\left(\alpha_{1}, \ldots, \alpha_{m}\right) \Omega, \quad \text { for all } \alpha_{1}, \ldots, \alpha_{m} \in \Omega^{1}(M) .
$$

From (5), we obtain that $\mathcal{L}_{\tilde{\#}_{(\Lambda, E)}(\delta f, f)} \Omega=\left(\operatorname{div}_{\Omega} \#_{\Lambda}(\delta f)+f \operatorname{div}_{\Omega} E+E(f)\right) \Omega$ and, using (2) and (12), we deduce that

$$
d^{(\Lambda, E)}\left(i(\delta f, f)\left(0, V_{\Omega}\right)\right)=-\left(\operatorname{div}_{\Omega} \#_{\Lambda}(\delta f)-m E(f)+f \operatorname{div}_{\Omega} E\right)\left(0, V_{\Omega}\right) .
$$

Thus, from (59), (60) and (61), it follows that

$$
\mathcal{M}_{T^{*} M \times \mathbb{R}}=2 \mathcal{M}_{(\Lambda, E)}+(m+1)[(E, 0)],
$$

where $\mathcal{M}_{T^{*} M \times \mathbb{R}}$ (respectively, $\left.\mathcal{M}_{(\Lambda, E)}\right)$ is the modular class of the Lie algebroid $\left(T^{*} M \times\right.$ $\left.\mathbb{R}, \llbracket, \rrbracket_{(\Lambda, E)}, \tilde{\#}_{(\Lambda, E)}\right)$ (respectively, of the Jacobi manifold $M$ ).

Now, suppose that $\left((A, \llbracket, \rrbracket, \rho), \phi_{0}, P\right)$ is a triangular generalized Lie bialgebroid of rank $n$ over an orientable manifold $M$ of dimension $m$. Let $\nu \in \Gamma\left(\wedge^{n} A^{*}\right)$ be a nowhere vanishing $n$-section and $\Omega \in \Omega^{m}(M)$ a volume form. We consider the section $V_{\nu}$ of $\wedge^{n} A \rightarrow M$ characterized by the relation

$$
\alpha_{1} \wedge \ldots \wedge \alpha_{n}=V_{\nu}\left(\alpha_{1}, \ldots, \alpha_{n}\right) \nu, \quad \text { for } \alpha_{1}, \ldots, \alpha_{n} \in \Gamma\left(A^{*}\right) .
$$

A direct computation proves that

$$
\left(\operatorname{div}_{V_{\nu}} \alpha\right) \nu=-\llbracket \alpha, \nu \rrbracket_{*}, \quad \text { for } \alpha \in \Gamma\left(A^{*}\right) .
$$

Therefore, using (56), (61) and (62), we conclude that

$$
\#_{P}\left(\mathcal{M}_{A}^{(\nu, \Omega)}\right)=-\mathcal{M}_{A^{*}}^{\left(V_{\nu}, \Omega\right)}+\Psi=\mathcal{M}_{\left(A, \phi_{0}, P\right)}^{\nu}-(n-1) X_{0}-\Psi^{\prime},
$$

where $\Psi$ and $\Psi^{\prime}$ are the 1-cocycles of the Lie algebroid $(A, \llbracket, \rrbracket, \rho)$ characterized by the relations

$$
\alpha(\Psi) \nu=\mathcal{L}_{\#_{P}(\alpha)} \nu+\llbracket \alpha, \nu \rrbracket_{*}, \quad \alpha\left(\Psi^{\prime}\right)=\operatorname{div}_{\Omega} \rho\left(\#_{P}(\alpha)\right)+i(P)(d \alpha),
$$

for all $\alpha \in \Gamma\left(A^{*}\right)$.

From (63), we obtain that

$$
\#_{P}\left(\mathcal{M}_{A}\right)=-\mathcal{M}_{A^{*}}+[\Psi]=\mathcal{M}_{\left(A, \phi_{0}, \rho\right)}-(n-1)\left[X_{0}\right]-\left[\Psi^{\prime}\right]
$$

$\#_{P}: H^{1}(A) \rightarrow H^{1}\left(A^{*}\right)$ being the homomorphism between $H^{1}(A)$ and $H^{1}\left(A^{*}\right)$ induced by the map $\#_{P}: \Gamma\left(A^{*}\right) \rightarrow \Gamma(A)$ (see Proposition 4.3 ). 
Finally, if $(\Lambda, E)$ is the Jacobi structure induced by the triangular generalized bialgebroid $\left(A, \phi_{0}, P\right)$ over the base manifold $M$, using (65), we conclude that

$$
\left(\rho, \phi_{0}\right)\left(\#_{P}\left(\mathcal{M}_{A}\right)\right)=\left(\rho, \phi_{0}\right)\left(\mathcal{M}_{\left(A, \phi_{0}, P\right)}\right)-\mathcal{M}_{(\Lambda, E)}+(n-m-1)[(E, 0)],
$$

where $\left(\rho, \phi_{0}\right): H^{1}\left(A^{*}\right) \rightarrow H_{L J}^{1}(M, \Lambda, E)$ is the homomorphism in cohomology induced by the map $\left(\rho, \phi_{0}\right): \Gamma(A) \rightarrow \Gamma(T M \times \mathbb{R}) \cong \mathfrak{X}(M) \oplus C^{\infty}(M, \mathbb{R})$ (see Corollary 4.5).

\subsection{Examples}

1. Triangular Lie bialgebroids. Let $(A, P)$ be a triangular Lie bialgebroid over $M$ in the sense of $[\mathrm{MX} 1]$, that is, $(A, \llbracket, \rrbracket, \rho)$ is a Lie algebroid and $P \in \Gamma\left(\wedge^{2} A\right)$ satisfies $\llbracket P, P \rrbracket=0$. Then, $\left(A, \phi_{0}=0, P\right)$ is a triangular generalized Lie bialgebroid and the dual bundle $A^{*} \rightarrow M$ to $A$ admits a Lie algebroid structure $\left(\llbracket, \rrbracket{ }_{*}, \rho_{*}\right)$ given by (27).

In this case, $X_{0}=-\#_{P}\left(\phi_{0}\right)=0$, the base space $M$ is a Poisson manifold, the cohomology operators $d_{*}$ and $d_{* X_{0}}$ coincide and $H^{*}\left(A^{*}\right)=H_{X_{0}}^{*}\left(A^{*}\right)$. Moreover, the homology operator $\mathfrak{d}: \Gamma\left(\wedge^{*} A^{*}\right) \rightarrow \Gamma\left(\wedge^{*-1} A^{*}\right)$ defined as in (39) is just $\mathfrak{d}=i(P) d-$ $d i(P)=[i(P), d]$.

If $n$ is the rank of $A$, the flat $A^{*}$-connection on $\wedge^{n} A^{*} \rightarrow M$ associated with $\mathfrak{d}$ is given by $\nabla_{\alpha} \Phi=\alpha \wedge d i(P) \Phi$, for $\alpha \in \Gamma\left(A^{*}\right)$ and $\Phi \in \Gamma\left(\wedge^{n} A^{*}\right)$ (see (49)).

Now, assume that $A$ is orientable and that $\nu \in \Gamma\left(\wedge^{n} A^{*}\right)$ is a nowhere vanishing $n$-section. Then, the section $\mathcal{M}_{(A, 0, P)}^{\nu} \in \Gamma(A)$, which we will denote by $\mathcal{M}_{(A, P)}^{\nu}$, is characterized by the condition (see $(56)$ )

$$
\alpha\left(\mathcal{M}_{(A, P)}^{\nu}\right) \nu=\mathcal{L}_{\# P(\alpha)} \nu+i(P)(d \alpha) \nu
$$

for $\alpha \in \Gamma\left(A^{*}\right)$. The cohomology class $\mathcal{M}_{(A, P)}=\left[\mathcal{M}_{(A, P)}^{\nu}\right] \in H^{1}\left(A^{*}\right)$ is just the modular class of the triangular Lie bialgebroid $(A, P)$ introduced in [Ko2]. Furthermore, from Theorem 4.10, it follows that if $\mathcal{M}_{(A, P)}$ is zero then $H^{k}\left(A^{*}\right) \cong H_{n-k}\left(A^{*}, \nabla\right)$, for all $k$. This result was also proved in [Ko2].

Next, we will consider some particular examples.

1a) Poisson manifolds. Let $\Lambda$ be a Poisson structure on a manifold $M$. If we consider the trivial Lie algebroid structure $([],, I d)$ on $T M$ then the pair $(T M, \Lambda)$ is a triangular Lie bialgebroid. Moreover, the corresponding Lie algebroid structure on $T^{*} M$ is the one associated with the Poisson structure $\Lambda$, that is, $\llbracket, \rrbracket_{*}=\llbracket, \rrbracket_{\Lambda}$ and $\rho_{*}=\#_{\Lambda}$. Thus, $H^{*}\left(T^{*} M\right)$ is the Lichnerowicz-Poisson cohomology $H_{L P}^{*}(M, \Lambda)$ of $M$.

On the other hand, in this case, $\mathfrak{d}$ is just the homology operator of the canonical homology complex of $M$ and $H_{*}\left(T^{*} M, \nabla\right)$ is the canonical homology $H_{*}^{c a n}(M, \Lambda)$ of $M$.

Furthermore, if $M$ is orientable, the modular class of $(T M, \Lambda)$ is just the well-known modular class of the Poisson manifold $(M, \Lambda)$ (see (56) and [W]). As a consequence, we deduce the following result (which it was proved in [BZ, ELW, X]): if the modular class of $M$ vanishes then $H_{L P}^{k}(M, \Lambda) \cong H_{n-k}^{c a n}(M, \Lambda)$, for all $k$.

1b) Triangular Lie bialgebras. Let $\left(\mathfrak{g},[,]^{\mathfrak{g}}\right)$ be a real Lie algebra of dimension $n$ and let $r$ be a solution of the classical Yang-Baxter equation on $\mathfrak{g}$, that is, $r \in \wedge^{2} \mathfrak{g}$ and $[r, r]^{\mathfrak{g}}=0$, where $[,]^{\mathfrak{g}}$ is the algebraic Schouten bracket of $\mathfrak{g}$ or, in other words, $[,]^{\mathfrak{g}}$ is the Schouten bracket of the Lie algebroid $\mathfrak{g} \rightarrow\{$ point $\}$. The 2 -vector $r$ can be viewed as an algebraic Poisson structure on $\mathfrak{g}$. 
Using this algebraic Poisson structure, one can introduce the homology complex

$$
\ldots \rightarrow \wedge^{k+1} \mathfrak{g}^{*} \stackrel{\mathfrak{d}}{\rightarrow} \wedge^{k} \mathfrak{g}^{*} \stackrel{\mathfrak{o}}{\rightarrow} \wedge^{k-1} \mathfrak{g}^{*} \rightarrow \ldots,
$$

where $\mathfrak{d}$ is the operator given by $\mathfrak{d}=i(r) \circ d-d \circ i(r)$. The resultant homology $H_{*}^{\text {can }}(\mathfrak{g}, r)$ is the algebraic canonical homology of $\mathfrak{g}$ associated with $r$.

The condition $[r, r]^{\mathfrak{g}}=0$ also implies that the operator $\sigma: \wedge^{k} \mathfrak{g} \rightarrow \wedge^{k+1} \mathfrak{g}$ given by

$$
\sigma(Q)=-[r, Q]^{\mathfrak{g}},
$$

is of square zero. Thus, we have the cohomology complex

$$
\ldots \rightarrow \wedge^{k-1} \mathfrak{g} \stackrel{\sigma}{\rightarrow} \wedge^{k} \mathfrak{g} \stackrel{\sigma}{\rightarrow} \wedge^{k+1} \mathfrak{g} \rightarrow \ldots
$$

The corresponding cohomology $H_{P}^{*}(\mathfrak{g}, r)$ is the algebraic Poisson cohomology of $\mathfrak{g}$ associated with $r$.

On the other hand, since the pair $(\mathfrak{g}, r)$ is a triangular Lie bialgebroid, the dual space $\mathfrak{g}^{*}$ admits a Lie bracket $[,]^{\mathfrak{g}^{*}}$ defined by (27). Moreover, if $d_{*}$ is the algebraic differential of $\left(\mathfrak{g}^{*},[,]^{\mathfrak{g}^{*}}\right)$ then $d_{*}=\sigma$ (see Proposition 4.2) and therefore $H^{*}\left(\mathfrak{g}^{*}\right)=H_{P}^{*}(\mathfrak{g}, r)$.

Now, denote by $\mathcal{M}_{\mathfrak{g}} \in \mathfrak{g}^{*}$ the modular character of $\left(\mathfrak{g},[,]^{\mathfrak{g}}\right)$ and by $\mathcal{M}_{(\mathfrak{g}, r)} \in \mathfrak{g}$ the modular class of the triangular Lie bialgebroid $(\mathfrak{g}, r) . \mathcal{M}_{\mathfrak{g}}$ is the modular class of the Lie algebroid $\mathfrak{g} \rightarrow$ \{point $\}$ (see [ELW]). Therefore, using (63) and (64), we can prove that $\mathcal{M}_{(\mathfrak{g}, r)}=\#_{r}\left(\mathcal{M}_{\mathfrak{g}}\right)+\xi$, where $\#_{r}: \mathfrak{g}^{*} \rightarrow \mathfrak{g}$ is the homomorphism induced by $r$ and $\xi \in \mathfrak{g}$ is characterized by the condition $\phi(\xi)=i(r)(d \phi)$, for all $\phi \in \mathfrak{g}^{*}$. Consequently, if $\#_{r}\left(\mathcal{M}_{\mathfrak{g}}\right)=-\xi$ then we deduce that $H_{P}^{k}(\mathfrak{g}, r) \cong H_{n-k}^{c a n}(\mathfrak{g}, r)$, for all $k$. A particular example of the above situation is the following one.

Let $\left(\mathfrak{u}(2),[,]^{\mathfrak{u}(2)}\right)$ be the Lie algebra of the unitary group $U(2)$. Then, there exists a basis $\{X, Y, Z, T\}$ of $\mathfrak{u}(2)$ such that $T$ belongs to the center of $\mathfrak{u}(2)$ and

$$
[X, Y]^{\mathfrak{u}(2)}=Z, \quad[X, Z]^{\mathfrak{u}(2)}=-Y, \quad[Y, Z]^{\mathfrak{u}(2)}=X .
$$

Thus, the 2-vector $r \in \wedge^{2} \mathfrak{g}$ given by $r=X \wedge T$ satisfies $[r, r]^{\mathfrak{u}(2)}=0$. Therefore, since $\mathfrak{u}(2)$ is a unimodular Lie algebra and $i(r)(d \phi)=0$, for all $\phi \in \mathfrak{u}(2)^{*}$, we conclude that

$$
H_{P}^{k}(\mathfrak{u}(2), r) \cong H_{4-k}^{c a n}(\mathfrak{u}(2), r), \text { for all } k .
$$

On the other hand, a direct computation shows that

$$
\begin{gathered}
H_{P}^{i}(\mathfrak{u}(2), r) \cong H_{4-i}^{\text {can }}(\mathfrak{u}(2), r) \cong \mathbb{R}, \text { for } i=0,4, \\
H_{P}^{j}(\mathfrak{u}(2), r) \cong H_{4-j}^{\text {can }}(\mathfrak{u}(2), r) \cong \mathbb{R}^{2}, \text { for } j=1,2,3 .
\end{gathered}
$$

2. Jacobi manifolds. Let $(M, \Lambda, E)$ be a Jacobi manifold of dimension $n$. For the dual Lie algebroid $\left(T^{*} M \times \mathbb{R}, \llbracket, \rrbracket_{(\Lambda, E)}, \widetilde{\#}_{(\Lambda, E)}\right)$ associated with the triangular generalized Lie bialgebroid $((T M \times \mathbb{R},[],, \pi),(0,1),(\Lambda, E))$, the cohomology operator $d_{*}$ (respectively, $d_{*(-E, 0)}$ ) is just the LJ-cohomology operator $d^{(\Lambda, E)}$ (respectively, the 1differentiable Chevalley-Eilenberg cohomology operator $\left.\left(d^{(\Lambda, E)}\right)_{(-E, 0)}\right)$ described in (12) (respectively, (21)).

On the other hand, in this case, the homology operator $\mathfrak{d}$ (see (39)) associated with the flat $\left(T^{*} M \times \mathbb{R}\right)$-connection $\nabla$ given by $(49)$ is just the homology operator $\partial^{(\Lambda, E)}$ introduced by Vaisman in [V3] (see (17)). Moreover, the modular class of the triangular 
generalized Lie bialgebroid $((T M \times \mathbb{R},[],, \pi),(0,1),(\Lambda, E))$ is the modular class of the Jacobi manifold $(M, \Lambda, E)$ (see Section 4.3).

Therefore, for unimodular Jacobi manifolds (i.e., with vanishing modular class) we have that

$$
\begin{gathered}
H_{L J}^{k}(M, \Lambda, E) \cong H_{n+1-k}\left(T^{*} M \times \mathbb{R}, \nabla\right), \\
H_{1-\operatorname{diff}}^{k}(M, \Lambda, E) \cong H_{n+1-k}\left(T^{*} M \times \mathbb{R}, \nabla^{(-E, 0)}\right),
\end{gathered}
$$

where $H_{L J}^{*}(M, \Lambda, E)$ (respectively, $\left.H_{1-\operatorname{diff}}^{*}(M, \Lambda, E)\right)$ is the LJ-cohomology (respectively, the 1-differentiable Chevalley-Eilenberg cohomology) of $M$ and $\nabla^{(-E, 0)}$ is the flat $\left(T^{*} M \times\right.$ $\mathbb{R})$-connection given by

$$
\nabla_{(\alpha, f)}^{(-E, 0)}(0, \Phi)=\nabla_{(\alpha, f)}(0, \Phi)+(\alpha, f) \wedge i(-E, 0)(0, \Phi)
$$

for $(\alpha, f) \in \Omega^{1}(M) \oplus C^{\infty}(M, \mathbb{R})$ and $(0, \Phi) \in \Gamma\left(\wedge^{n+1}\left(T^{*} M \times \mathbb{R}\right)\right) \cong\{0\} \oplus \Omega^{n}(M)$.

$H_{*}\left(T^{*} M \times \mathbb{R}, \nabla\right)$ is the canonical LJ-homology (respectively, the Jacobi homology) of $M$ in the terminology of [LLMP] (respectively, [V3]) and the isomorphism in (67) was obtained by Vaisman in [V3].

Examples of Jacobi manifolds such that its modular class vanishes (respectively, does not vanish) can be found in [LLMP, V3].

\section{References}

[BV] K. H. Bhaskara and K. Viswanath, Poisson algebras and Poisson manifolds, Research Notes in Math. 174, Pitman, London, 1988.

[B] J. L. Brylinski, A differential complex from Poisson manifolds, J. Differential Geometry 28 (1988), 93-114.

[BZ] J. L. Brylinski and G. Zuckerman, The outer derivation of a complex Poisson manifold, J. Reine Angew. Math. 506 (1999), 181-189.

[CE] H. Cartan and S. Eilenberg, Homological Algebra, Princeton Univ. Press, Princeton, NJ, 1973.

[D] V. G. Drinfeld, Hamiltonian Lie groups, Lie bialgebras and the geometric meaning of the classical Yang-Baxter equation, Sov. Math. Doklady 27 (1983), 68-71.

[ELW] S. Evens, J.-H. Lu and A. Weinstein, Transverse measures, the modular class and a cohomology pairing for Lie algebroids, Quart. J. Math. Oxford Ser. 50 (1999), 417-436.

[F] B. Fuchssteiner, The Lie algebra structure of degenerate Hamiltonian and biHamiltonian systems, Progr. Theoret. Phys. 68 (1982), 1082-1104.

[GL] F. Guédira and A. Lichnerowicz, Géométrie des algèbres de Lie locales de Kirillov, J. Math. Pures Appl. 63 (1984), 407-484.

[IM1] D. Iglesias and J. C. Marrero, Some linear Jacobi structures on vector bundles, C. R. Acad. Sci. Paris Sér. I 331 (2000), 125-130.

[IM2] D. Iglesias and J. C. Marrero, Generalized Lie bialgebroids and Jacobi structures, J. Geom. Phys. 40 (2001), 176-200.

[IM3] D. Iglesias and J. C. Marrero, Generalized Lie bialgebras and Jacobi structures on Lie groups, Preprint (2001), arXiv: math.DG /0102171.

[KS] Y. Kerbrat and Z. Souici-Benhammadi, Variétés de Jacobi et groupoïdes de contact, C. R. Acad. Sci. Paris Sér. I 317 (1993), 81-86. 
[Ki] A. Kirillov, Local Lie algebras, Russian Math. Surveys 31 (1976), 55-75.

[Ko1] Y. Kosmann-Schwarzbach, Exact Gerstenhaber algebras and Lie bialgebroids, Acta Appl. Math. 41 (1995), 153-165.

[Ko2] Y. Kosmann-Schwarzbach, Modular vector fields and Batalin-Vilkovisky algebras, in: Poisson Geometry, Banach Center Publ. 51, 2000, 109-129.

[K] J. L. Koszul, Crochet de Schouten-Nijenjuis et cohomologie, in: Elie Cartan et les Math. d'Aujourd'hui, Astérisque, hors série (1985), 251-271.

[LLMP] M. de León, B. López, J. C. Marrero and E. Padrón, Lichnerowicz-Jacobi cohomology and homology of Jacobi manifolds: modular class and duality, Preprint (1999), arXiv: math.DG/9910079.

[LMP] M. de León, J. C. Marrero and E. Padrón, On the geometric quantization of Jacobi manifolds, J. Math. Phys. 38 (12) (1997), 6185-6213.

[L1] A. Lichnerowicz, Les variétés de Poisson et leurs algèbres de Lie associées, J. Differential Geometry 12 (1977), 253-300.

[L2] A. Lichnerowicz, Les variétés de Jacobi et leurs algèbres de Lie associées, J. Math. Pures Appl. 57 (1978), 453-488.

[M] K. Mackenzie, Lie Groupoids and Lie Algebroids in Differential Geometry, Cambridge University Press, 1987.

[MX1] K. Mackenzie and P. Xu, Lie bialgebroids and Poisson groupoids, Duke Math. J. 73 (1994), 415-452.

[MX2] K. Mackenzie and P. Xu, Integration of Lie bialgebroids, Topology 39 (2000), 445-467.

[MMP] J. C. Marrero, J. Monterde and E. Padrón, Jacobi-Nijenhuis manifolds and compatible Jacobi structures, C. R. Acad. Sci. Paris Sér. I 329 (1999), 797-802.

[N] Ngô-van-Quê, Sur l'espace de prolongement différentiable, J. Differential Geometry 2 (1968), 33-40.

[P] J. Pradines, Théorie de Lie pour les groupoïdes différentiables. Calcul différentiel dans la catégorie des groupoïdes infinitésimaux, C. R. Acad. Sci. Paris Sér. A 264 (1967), $245-248$.

[V1] I. Vaisman, Remarkable operators and commutation formulas on locally conformal Kähler manifolds, Compositio Math. 40 (1980), 287-299.

[V2] I. Vaisman, Lectures on the Geometry of Poisson Manifolds, Progress in Math. 118, Birkhäuser, Basel, 1994.

[V3] I. Vaisman, The BV-algebra of a Jacobi manifold, Ann. Polon. Math. 73 (2000), 275-290.

[W] A. Weinstein, The modular automorphism group of a Poisson manifold, J. Geom. Phys. 23 (1997), 379-394.

[X] P. Xu, Gerstenhaber algebras and BV-algebras in Poisson geometry, Comm. Math. Phys. 200 (1999), 545-560. 